The University of San Francisco

USF Scholarship: a digital repository @ Gleeson Library |

Geschke Center

2013

\title{
The Escape Fraction of Ionizing Radiation from Galaxies
}

\author{
A Benson \\ Aparna Venkatesan \\ University of San Francisco, avenkatesan@usfca.edu \\ J Shull
}

Follow this and additional works at: http://repository.usfca.edu/phys

Part of the Astrophysics and Astronomy Commons, and the Physics Commons

\section{Recommended Citation}

Benson, A., Venkatesan, A., \& Shull, J. (2013). The escape fraction of ionizing radiation from galaxies. Astrophysical Journal, 770(1), 76. doi:10.1088/0004-637X/770/1/76

This Article is brought to you for free and open access by the College of Arts and Sciences at USF Scholarship: a digital repository @ Gleeson Library | Geschke Center. It has been accepted for inclusion in Physics and Astronomy by an authorized administrator of USF Scholarship: a digital repository @ Gleeson Library | Geschke Center. For more information, please contact repository@usfca.edu. 


\title{
THE ESCAPE FRACTION OF IONIZING RADIATION FROM GALAXIES
}

\author{
Andrew Benson ${ }^{1}$, Aparna VenKatesan ${ }^{2}$, and J. Michael Shull ${ }^{3}$ \\ ${ }^{1}$ Carnegie Observatories, 813 Santa Barbara Street, Pasadena, CA 91101, USA; abenson@ obs.carnegiescience.edu \\ 2 Department of Physics and Astronomy, University of San Francisco, San Francisco, CA 94117, USA; avenkatesan@ usfca.edu \\ ${ }^{3}$ CASA, Department of Astrophysical and Planetary Sciences, University of Colorado, Boulder, CO 80309, USA; michael.shull@ colorado.edu \\ Received 2012 December 18; accepted 2013 April 22; published 2013 May 24
}

\begin{abstract}
The escape of ionizing radiation from galaxies plays a critical role in the evolution of gas in galaxies, and the heating and ionization history of the intergalactic medium. We present semi-analytic calculations of the escape fraction of ionizing radiation for both hydrogen and helium from galaxies ranging from primordial systems to disk-type galaxies that are not heavily dust-obscured. We consider variations in the galaxy density profile, source type, location, and spectrum, and gas overdensity/distribution factors. For sufficiently hard first-light sources, the helium ionization fronts closely track or advance beyond that of hydrogen. Key new results in this work include calculations of the escape fractions for He I and He II ionizing radiation, and the impact of partial ionization from Xrays from early active galactic nuclei or stellar clusters on the escape fractions from galaxy halos. When factoring in frequency-dependent effects, we find that X-rays play an important role in boosting the escape fractions for both hydrogen and helium, but especially for He II. We briefly discuss the implications of these results for recent observations of the He II reionization epoch at low redshifts, as well as the UV data and emission-line signatures from early galaxies anticipated from future satellite missions.
\end{abstract}

Key words: cosmology: theory - dark ages, reionization, first stars

Online-only material: color figures

\section{INTRODUCTION}

The escape fraction of ionizing radiation, $f_{\text {esc }}$, is a key quantity in the feedback from galaxies on the intergalactic medium (IGM) at all redshifts, $z$, and is an important input parameter in reionization models and cosmological simulations. It is typically defined as the fraction of hydrogen (or when relevant, helium) ionizing photons that escape from a source galaxy to the IGM after accounting for recombinations within the galaxy and its halo. Such radiation profoundly affects the thermal and ionization history of the IGM and is an important factor in the modeling of many astrophysical problems, ranging from metal ionization states in absorber systems to the reionization of the IGM (Loeb \& Barkana 2001). The converse problem - that of trapped radiation in galaxies-is also important for understanding the viability of detecting primordial galaxies or Ly $\alpha$ emitters through emission-line and other signatures (Tumlinson et al. 2001; Rhoads et al. 2004).

Theoretical studies (Dove et al. 2000; Wood \& Loeb 2000; Ricotti \& Shull 2000; Ricotti et al. 2002; Gnedin et al. 2008; Wise \& Cen 2009; Razoumov \& Sommer-Larsen 2010; Yajima et al. 2011; Fernandez \& Shull 2011) have indicated a wide range of values for $f_{\text {esc }}$ for hydrogen, ranging from $1 \%-100 \%$, beginning with the first detailed studies of the Galaxy's diffuse ionizing gas by Dove \& Shull (1994). Owing to the complexity and variety of factors that influence photon escape, any single theoretical trend must necessarily be seen as only one aspect of reality. These factors include galaxy mass and morphology, galaxy redshift, gas density profiles, composition, source luminosities/ spectra, source distribution and time evolution within the galaxy, and cloud mass/distribution factors in the interstellar medium (ISM). Several numerical simulation studies have recently found that $f_{\text {esc }}$ increases with decreasing galaxy mass, and with galaxy redshift (Gnedin et al. 2008; Shull et al. 2012a). Higher mass galaxies will have more gas to ionize (leading to lower $f_{\text {esc }}$ ), but owing to their deeper potential wells, they are also more likely to have higher star formation rates and more collisionally ionized gas in their ISM (leading overall to higher $f_{\text {esc }}$, even if the sources are embedded in neutral or molecular gas). Similar complexities arise in redshift studies of $f_{\text {esc }}$ : in principle, the abundance of lower-mass galaxies and higher star formation rates at high redshifts should lead to higher values of the escape fraction. Some authors find higher values of $f_{\text {esc }}$ for dwarf galaxies and Ly $\alpha$ emitters at high redshifts relative to massive galaxies at similar or lower redshifts, but these are complicated by model assumptions underlying these rather different systems (Ricotti \& Shull 2000; Wood \& Loeb 2000; Gnedin et al. 2008). Direct comparisons with data of low-redshift systems will provide key insights into the many convolved factors, but this has been hindered by a conspicuous lack of numerical simulations of $f_{\text {esc }}$ for low $-z$ systems. In addition, values of $f_{\text {esc }}$ that exceed $20 \%$ in low-mass galaxies are possibly needed to explain the observational constraints on completing hydrogen reionization by $z \simeq 7$ (Shull et al. 2012a; Robertson et al. 2013; Ellis et al. 2013; Schenker et al. 2013; McLure et al. 2013; Dunlop et al. 2012; Finkelstein et al. 2012). The results of the many theoretical studies, as well as various factors and trends, are summarized comprehensively in Fernandez \& Shull (2011).

Observationally, the constraints for $f_{\text {esc }}$ have so far been for $\mathrm{H}$ alone, focusing (through various techniques) on measuring the escape of Lyman-continuum (LyC) radiation ( $h v \geqslant 1$ Ryd). Here, there has been a gradual approach to some consensus values, and an indication from the past few years of data that $f_{\text {esc }, \mathrm{H}}$ increases with increasing redshift, confirming one of the theoretical predictions stated above. Although observations about a decade ago (Steidel et al. 2001) of LyC emission from Lyman-break galaxies (LBGs) at $z \sim 3$ indicated high values of $f_{\text {esc }, \mathrm{H}} \gtrsim 0.5$, more recent analyses of these objects at similar or lower redshifts (Shapley et al. 2006; Siana et al. 2010; Nestor et al. 2011) have derived lower values of $\sim 0.1-0.25$, with only 
$\sim 10 \%$ of LBGs and Ly $\alpha$ emitters exhibiting large $f_{\text {esc }}$ values. Note that these $f_{\mathrm{esc}, \mathrm{H}}$ values are a measurement of the relative escape fraction, the ratio of fractions of escaping ionizing to non-ionizing UV photons, as defined by these authors. There are also indications from measurements of the UV luminosity density of galaxy samples at $z \sim 6-8$ that $f_{\text {esc }, \mathrm{H}}$ may exceed $50 \%$ (Finkelstein et al. 2012), in agreement with Shull et al. (2012a) and with simulations finding increasing $f_{\text {esc }}$ values toward the faint end of the galaxy luminosity function (Ciardi et al. 2012). At lower redshifts $(z<3)$, observations have indicated $f_{\text {esc }, \mathrm{H}} \sim 0.01-0.1$ from high-mass galaxies (Leitherer et al. 1995; Deharveng et al. 2001; Heckman et al. 2011; Grimes et al. 2009). Although these fractions appear significantly lower than the $z \sim 3$ values at first, at least some of these systems (Heckman et al. 2011) exhibit values of about 30\%-40\% when recast in terms of the relative escape fraction. Again, it would be valuable to have specific theoretical constraints for comparison, but the field has had very few numerical simulations of $f_{\text {esc }}$ at low redshifts.

We note that this relative escape fraction is arguably more comparable to the results of this work (and many others cited above) than the absolute escape fraction. The absolute escape fraction includes, by definition, the effects of any dust obscuration. However, compared to hydrogen photoelectric absorption, dust is usually unimportant for the EUV (LyC). Our models do not at present consider the effects of dust, as they deal with primordial gas. By considering the relative escape fraction (usually the ratio of escape fractions at $900 \AA$ and $1500 \AA$ ) the effects of dust are mitigated, since the obscuration due to dust should be the same at the two wavelengths modulo the difference in wavelength-dependent opacity. ${ }^{4}$ Our main goals here in this work are to model a variety of galaxies that are not heavily obscured by dust, ranging from primordial systems that are more prevalent at high redshifts to the more disk-like galaxies typical at intermediate to low redshifts.

In contrast to the case of hydrogen, there are no data and detailed theoretical studies of the relevant escape fraction for radiation capable of ionizing $\mathrm{He}$ II $(h v \geqslant 4 \mathrm{Ryd}), f_{\text {esc,He. In }}$ this paper, we derive the values and geometry of the escape of helium ionizing radiation from the first galaxies. This quantity can have a profound influence on the thermal and ionization conditions in the IGM and be an important input for future IGM and cosmic microwave background studies, as well as searches for the first stellar clusters. We find that the hardness of the source spectrum is an important factor in determining the relative ionization-front (hereafter, "I-front") evolution, and we consider both active galactic nuclei (AGNs)/QSOs as well as metal-free stellar populations which, unlike their low-metallicity counterparts, produce helium ionizing radiation. We also consider the role played by X-rays in the escape of ionizing radiation from early galaxies, particularly for He. We present our model and assumptions in Section 2, and the role of frequency-dependent factors in the relative propagation of $\mathrm{H}$ and $\mathrm{He}$ I-fronts in Section 3. We present our results and detailed calculations in Section 4, allowing for variations in galaxy density profile, source spectrum, source location, and gas cloud factors, and we conclude in Section 5.

\footnotetext{
4 The difference in opacity between $1500 \AA$ and $1000 \AA$ may typically be a factor of 1.85 for the standard ISM extinction curve. Future theoretical studies of escape fractions should attempt to incorporate the effects of dust and predict relative escape fractions directly. See Benson et al. (2001) for an example of escape fraction calculations which do account for the effects of dust.
}

\section{BACKGROUND AND MODEL}

Our goal is to compute the emergent spectrum, ${ }^{5} S_{R_{\mathrm{vir}}}(v)$, of ionizing photons emitted by some source (a star cluster or AGN) with intrinsic spectrum $S_{0}(v)$ which is embedded within the ISM of a galaxy and/or a more extended gaseous halo. Throughout this work we consider a point source emitting isotropically such that, in absence of absorption, the flux from the source will decline with distance as $1 / r^{2}$. When absorption is included we ignore scattering into and out of the line of sight to the source.

Given the emergent spectrum and the known input spectrum the escape fraction is defined as:

$$
f_{\mathrm{esc}}=\frac{\int_{\nu_{0}}^{\infty} S_{R_{\mathrm{vir}}}(v) d v}{\int_{v_{0}}^{\infty} S_{0}(v) d v},
$$

where $v_{0}$ is the frequency corresponding to the ionization potential for whichever species is of interest, with $h v_{0}=$ $13.6 \mathrm{eV}, 24.6 \mathrm{eV}$ and $54.4 \mathrm{eV}$ for $\mathrm{H}$ i, He $\mathrm{I}$ and $\mathrm{He}$ II respectively. We will consider the gaseous environment of the source to be a mixture of hydrogen and helium, and to have some density distribution $n_{i}(\mathbf{x})$ for species $i=\mathrm{H}$, He, with the density distributions for hydrogen and helium differing only by a constant proportionality factor appropriate for primordial abundances.

We consider a source located at position $\mathbf{x}_{0}$ within this density distribution. Along a direction $\hat{\mathbf{n}}$ from this source we consider the propagation of the input spectrum through the absorbing gas. We assume that at each distance, $d$, from the source the hydrogen and helium reach photoionization balance at a temperature $T_{0}$. Given the electron density, $n_{\mathrm{e}}$, the equations of photoionization balance imply that

$$
\begin{aligned}
& 0=\Sigma_{\mathrm{HI}_{\mathrm{I}}} n_{\mathrm{H}}\left(1-x_{\mathrm{HII}}\right)-\alpha_{\mathrm{HII}}^{(2)}\left(T_{0}\right) n_{\mathrm{e}} n_{\mathrm{H}} x_{\mathrm{HII}}, \\
& 0=\Sigma_{\mathrm{He} \text { I }} n_{\mathrm{He}}\left(1-x_{\mathrm{He} \mathrm{II}}-x_{\mathrm{He} \mathrm{III}}\right)-\alpha_{\mathrm{He} \text { I }}^{(2)}\left(T_{0}\right) n_{\mathrm{e}} n_{\mathrm{He}} x_{\mathrm{He} \mathrm{II}} \\
& 0=\Sigma_{\mathrm{He} \text { II }} n_{\mathrm{He}} x_{\mathrm{He} \text { II }}-\alpha_{\mathrm{He} \text { III }}^{(2)}\left(T_{0}\right) n_{\mathrm{e}} n_{\mathrm{He}} x_{\mathrm{He} \text { III }},
\end{aligned}
$$

where the densities are evaluated at position $\mathbf{x}_{0}+d \hat{\mathbf{n}}$, and $\Sigma_{i}$ is

$$
\Sigma_{i}=\int_{\nu_{0}}^{\infty} S(\nu) \sigma_{i}(\nu) d \nu
$$

Here, $\alpha_{i}^{(2)}(T)$ is the case-B recombination rate coefficient for ionization state $i, \sigma_{i}(v)$ is the photoionization cross-section for ionization state $i=\mathrm{HI}$, He I, He II, and $x_{i}$ is the fraction of each atomic species in ionization state $i$. Defining $\mathcal{R}_{i}=\Sigma_{i} / \alpha_{i+1}^{(2)} n_{\mathrm{e}}$, with the convention that

$$
i+1= \begin{cases}\mathrm{H}_{\mathrm{II}} & \text { for } i=\mathrm{H}_{\mathrm{I}} \\ \mathrm{He}_{\mathrm{II}} & \text { for } i=\mathrm{He}_{\mathrm{I}} \\ \mathrm{He}_{\mathrm{III}} & \text { for } i=\mathrm{He}_{\mathrm{II}}\end{cases}
$$

\footnotetext{
5 Typically, the emergent spectrum is defined as that at infinite distance from the source, but without including radiative transfer through the IGM. In cases where we consider density profiles that extend to infinity (and whose density falls only slowly with distance) we will define the emergent spectrum as that at the virial radius of the dark matter halo associated with the source. Our justification is that the virial radius marks the approximate boundary between galactic and intergalactic environment, and the escape fraction is usually thought of as the fraction of ionizing photons emitted by the source which arrive at the IGM.
} 
these equations have solution

$$
\begin{aligned}
x_{\mathrm{H} \mathrm{II}} & =\frac{\mathcal{R}_{\mathrm{H}_{\text {II }}}}{1+\mathcal{R}_{\mathrm{H} \text { II }}}, \\
x_{\mathrm{He} \text { II }} & =\frac{\mathcal{R}_{\mathrm{He} \mathrm{II}}}{1+\mathcal{R}_{\mathrm{He} \text { II }}+\mathcal{R}_{\mathrm{He} \text { II }} \mathcal{R}_{\mathrm{He} \mathrm{III}}}, \\
x_{\mathrm{He} \mathrm{III}} & =\frac{\mathcal{R}_{\mathrm{He} \text { II }} \mathcal{R}_{\mathrm{He} \mathrm{III}}}{1+\mathcal{R}_{\mathrm{He} \text { II }}+\mathcal{R}_{\mathrm{He} \text { II }} \mathcal{R}_{\mathrm{He} \mathrm{III}}} .
\end{aligned}
$$

We will assume a constant gas temperature of $T_{0}=10^{4} \mathrm{~K}$ throughout, and do not explicitly solve for the temperature. We do not expect the temperature within the I-fronts to be much below $10^{4} \mathrm{~K}$; in fact, photoheated primordial gas has temperatures $\gtrsim 20,000 \mathrm{~K}$, which has even been measured in the Ly $\alpha$ forest (Becker et al. 2011). For the $10^{8} M_{\odot}$ halo at $z=10$ that we will consider in Section 4.4 the virial temperature is $\sim 1.1 \times 10^{4} \mathrm{~K}$ (Donahue \& Shull 1991; Becker et al. 2011).

The ionization balance depends on the electron density, which can be found directly from the ionization states:

$$
n_{\mathrm{e}}=n_{\mathrm{H}} x_{\mathrm{HII}}+n_{\mathrm{He}}\left(x_{\mathrm{He} \text { II }}+2 x_{\mathrm{He} \text { III }}\right) .
$$

Therefore, we make an initial guess at the ionization states to allow us to compute $n_{\mathrm{e}}$ and then iteratively update the ionization fractions using the above equations until we obtain a converged solution for $n_{\mathrm{e}}$. After finding the ionization states we compute the optical depth to photoionization across a small increment in distance $\delta d$ using

$$
\delta \tau=\delta d \sum_{i} \int_{v_{0, i}}^{\infty} n_{i^{\prime}} x_{i} \sigma_{i}(\nu) d \nu
$$

where $i^{\prime}=\mathrm{H}$ for $i=\mathrm{H}$ I and $i^{\prime}=\mathrm{He}$ for $i=\mathrm{He}$ I or He II. The step in distance, $\delta d$ is chosen to ensure $\delta \tau \ll 1$ and $\delta d \ll r_{\mathrm{s}}$ where $r_{\mathrm{s}}$ is the characteristic length scale of the density profile being considered. The spectrum is then updated using

$$
S(v) \rightarrow S(v) \frac{\exp (-\delta \tau)}{(1+\delta d / d)^{2}},
$$

where the term in the numerator on the right-hand side (rhs) accounts for absorption of photons by the $\mathrm{H}$ and $\mathrm{He}$, and the term in the denominator accounts for the $1 / r^{2}$ reduction in flux with distance from the source. We repeat this process until a sufficiently large distance is reached that the spectrum is no longer changing significantly or, when considering source embedded in dark matter halos, the IGM (i.e., the halo virial radius) is reached. We then compute the escape fractions, $f_{i, \text { esc }}(\hat{\mathbf{n}})$, as defined above. The mean escape fraction, averaged over all directions, is then

$$
\left\langle f_{i, \mathrm{esc}}\right\rangle=\frac{1}{4 \pi} \int f_{i, \mathrm{esc}}(\hat{\mathbf{n}}) d \Omega .
$$

\subsection{Inclusion of Clouds}

To include clouds (i.e., regions of enhanced density) in our calculations we follow Fernandez \& Shull (2011). Clouds are defined by three quantities: their radius $\left(r_{\mathrm{c}}\right)$, their volume filling factor $\left(f_{\mathrm{c}}\right)$, and the cloud overdensity, $C$, the ratio of density in clouds to non-cloud regions (referred to as the "clumping factor" in Fernandez \& Shull 2011).
Along each direction, $\hat{\mathbf{n}}$, we construct a cylindrical volume with radius equal to the cloud radius such that any cloud whose center lies within this cylinder will intersect the line of sight along which the escape fraction is being computed. We find the mean number of clouds in this cylinder based on their radius and volume filling factor, $\left\langle N_{\mathrm{c}}\right\rangle=f_{\mathrm{c}} \pi r_{\mathrm{c}}^{2} d_{\max } /(4 \pi / 3) r_{\mathrm{c}}^{3}$, where $d_{\text {max }}$ is the maximum distance from the source to be considered. The actual number of clouds in the cylinder is then drawn from a Poisson distribution with this mean. Each cloud is located randomly within the cylinder and the section of the line of sight that the cloud intersects is determined.

In propagating the spectrum through this cloudy medium we now choose the steps in distance $\delta d$ such that cloud boundaries always precisely coincide with a step. Therefore any given step is either in a cloud or not in a cloud (i.e., a step is never partly in a cloud and partly not). In cloud regions the gas density is increased appropriately ${ }^{6}$ (see Fernandez \& Shull 2011). The emergent spectrum of photons then accounts for the effects of clouds along the line of sight.

\section{THE EVOLUTION OF HELIUM IONIZATION FRONTS}

\subsection{The Critical Spectral Index}

We begin by calculating the critical spectral index at which the rate of $\mathrm{H}$ and $\mathrm{He}$ photoionizations become equal. For a source spectrum whose specific luminosity $\left(\mathrm{erg} \mathrm{s}^{-1} \mathrm{~Hz}^{-1}\right.$ ) goes as $L_{v} \propto v^{-\alpha}$, we find that the ratio of photoionization rates per atom for $\mathrm{He}$ and $\mathrm{H}, X_{\mathrm{c}}$, is:

$$
X_{\mathrm{c}} \equiv \frac{Q(\mathrm{He} \mathrm{II}) / n_{\mathrm{He}}}{Q(\mathrm{HI}) / n_{\mathrm{H}}}=\left[\frac{\int_{4 \mathrm{Ryd} / h}^{\infty} d v L_{v} /(h v)}{\int_{1 \mathrm{Ryd} / h}^{\infty} d v L_{v} /(h v)}\right] \frac{n_{\mathrm{H}}}{n_{\mathrm{He}}} .
$$

The primordial helium mass fraction $Y_{\mathrm{He}} \approx 0.25$ (Peimbert et al. 2007; Aver et al. 2010), and the currently permitted range of $\Omega_{\mathrm{b}} h^{2}=0.02255 \pm 0.00054$ (Komatsu et al. 2011). This leads to a range of values for the relative helium fraction by number, $y=n_{\mathrm{He}} / n_{\mathrm{H}}=0.0789-0.0823$. We find that $X_{\mathrm{c}}$ is unity when:

$$
4^{-\alpha_{\text {crit }}}=0.08, \quad \text { or } \alpha_{\text {crit }}=-\frac{\ln (y)}{\ln (4)} \simeq 1.82 \pm 0.03 \text {, }
$$

if we assume $y=0.080 \pm 0.003$. Therefore, in the case of a source with $\alpha \lesssim 1.82$, the helium I-fronts may begin to overtake or coincide with the $\mathrm{H}$ I-fronts; such a hard spectral index may already be seen in $z \sim 0.03-1.45$ QSO spectra (Shull et al. 2012b) as we discuss below. For a source spectrum with multiple indices, e.g., $\alpha_{1}$ between $v_{1}$ and $v_{2}$, and $\alpha_{2}$ beyond $v_{2}$, Equation (14) is unity when:

$$
\frac{v_{2}^{\alpha_{2}}}{v_{1}^{\alpha_{1}}} \times \frac{\alpha_{1}}{\alpha_{2}} \times 4^{-\alpha_{2}}=0.08
$$

To extend this estimate to the case of metal-free stars, we now consider three cases involving metal-free initial mass functions (IMFs) in the mass ranges $1-100 M_{\odot}$ (Tumlinson et al. 2003), 10-140 $M_{\odot}$ (Tumlinson et al. 2004), and $\gtrsim 100 M_{\odot}$ (Bromm et al. 2001), all with a Salpeter slope unless noted otherwise. The first represents a roughly present-day IMF, the second the bestcase IMF which matches the current data on nucleosynthesis

\footnotetext{
6 It is possible for clouds to overlap. In such cases we still increase the density only by a factor of $C$ relative to the background, not by a factor of $2 C$ for example. This overlap means that the actual volume filling factor, $f_{\mathrm{c}}^{\prime}$, will be slightly smaller than the input filling factor, $f_{\mathrm{c}}: f_{\mathrm{c}}^{\prime}=1-\exp \left(-f_{\mathrm{c}}\right)$ on average. For the cases we consider, $f_{\mathrm{c}}=0.2$, so $f_{\mathrm{c}}^{\prime}=0.18$, a small difference.
} 


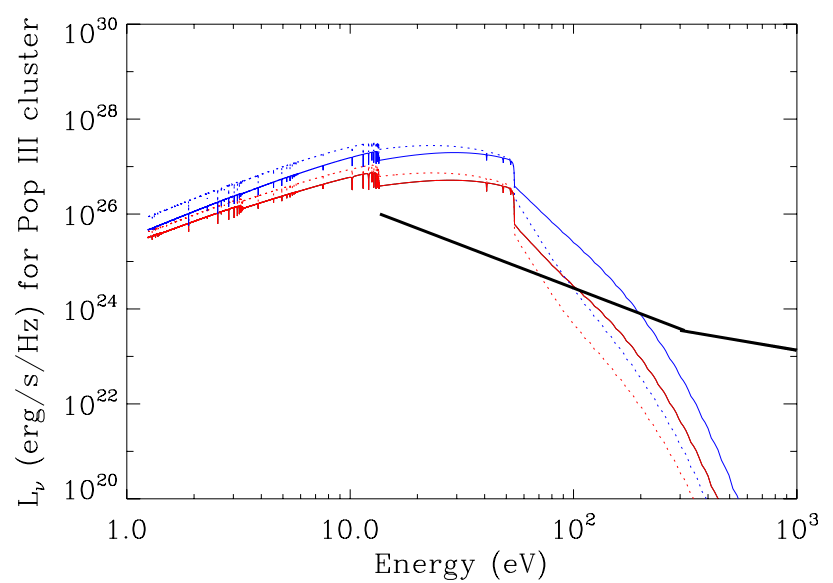

Figure 1. Specific luminosity as a function of energy of a fiducial $10^{6} M_{\odot}$ metal-free stellar cluster, based on Tumlinson et al. (2004). Red and blue lines represent $1-100 M_{\odot}$ and 10-140 $M_{\odot}$ IMFs, with solid and dashed lines in each case denoting the values on the ZAMS and at 2 Myr. Note the relative flatness (hardness) of the spectrum between the HI and He II ionizing thresholds at $13.6 \mathrm{eV}$ and $54.4 \mathrm{eV}$ respectively, and the rapid decline of the He ionizing flux with time. For comparison, a typical QSO spectrum (arbitrarily normalized) with slope $\alpha=1.8$ and 0.8 in the respective energy ranges $13.6-300 \mathrm{eV}$ and $\gtrsim 300 \mathrm{eV}$ is also shown.

(A color version of this figure is available in the online journal.)

and reionization, and the third, an IMF consisting of very massive stars. Such stars can lead to pair-instability supernovae in the mass range 140-260 $M_{\odot}$ and may dominate in primordial gas that lacks metal coolants. Thus $140 M_{\odot}$ divides stellar masses into two regimes that have similar radiative but different nucleosynthetic yield properties. For the first two of these IMFs, we display the specific intensity of a fiducial $10^{6} M_{\odot}$ zero-metal (Pop III) cluster as a function of energy in Figure 1. In each of these cases, two curves corresponding to the zero-age main sequence (ZAMS) and at times of 2 Myr are shown; for all the curves, we observe the relative hardness of the cluster's radiation between $1 \mathrm{Ryd}$ and $4 \mathrm{Ryd}$. A numerical evaluation of Equation (14) yields a value $X_{\mathrm{c}} \simeq 0.24(0.07)$ for the ZAMS (2 Myr) $1-100 M_{\odot}$ IMF, and $X_{\mathrm{c}} \simeq 0.43(0.11)$ for the ZAMS (2 Myr) $10-140 M_{\odot} \mathrm{IMF}$.

The fact that $X_{\mathrm{c}}$ never exceeds unity implies that the relative rate of $\mathrm{He}$ to $\mathrm{H}$ photoionizations remains below one, and therefore the He I-front evolution cannot exceed that of $\mathrm{H}$ for these IMFs. This is mostly due to the sharp fall off of the intensity beyond the He ionization threshold. For comparison, Figure 1 also shows the spectrum of a quasar (arbitrarily normalized) with slope $\alpha=1.8$ and 0.8 in the energy ranges $13.6-300 \mathrm{eV}$ and $\gtrsim 300 \mathrm{eV}$ respectively (Venkatesan et al. 2001). Such a source would clearly meet the criterion in Equation (15), and the $\mathrm{He}$ I-fronts could "keep up" with those associated with H. AGNs with sufficiently hard spectral indices are already observed in Cosmic Origins Spectrograph (COS) spectra of $z \sim 0.03-1.45$ QSOs (Shull et al. 2012b) where composite spectral slopes $\alpha=$ 1.4-1.5 are observed at energies $1.0-1.5 \mathrm{Ryd}$. This is a harder spectral slope than the $\alpha_{\text {crit }}$ derived earlier, if it continues to the 4 Ryd edge for He II reionization. However, this is within the range of the predicted critical spectral index when frequencydependent effects in the source spectrum are factored in, as well as the range detected observationally in low- $z$ AGNs with COS. We now show this in the next subsection.

\subsection{Frequency-dependent Effects in the Source Spectrum}

In order to compare the values of $f_{\text {esc }}$ for $\mathrm{H}$ and $\mathrm{He}$ for any general galaxy density profile, we begin with Equation (12) of
Dove \& Shull (1994),

$$
f_{\mathrm{esc}}=1-\frac{4 \pi \alpha_{\mathrm{B}}}{S_{0}} \int_{0}^{\infty} n_{\mathrm{e}} n_{\mathrm{H}} r^{2} d r .
$$

In order to assess the impact of frequency dependence in the input spectrum, we replace $S_{0}$ with a frequency-dependent function for the source photon luminosity, $S_{0}\left(v / v_{0}\right)^{-(\alpha+1)}$. Thus, the first part of the second term in the equation above becomes:

$$
\frac{4 \pi \alpha_{\mathrm{B}}}{\int d \nu S_{0}\left(\nu / \nu_{0}\right)^{-(\alpha+1)}} .
$$

The constant $S_{0}$ has to be evaluated through integration from a threshold energy, e.g., from $h v_{\mathrm{H}}=1$ Ryd or $h v_{\mathrm{He}}=4$ Ryd, up to infinite energy, and by setting the integral equal to the $\mathrm{H}$-ionizing (or He-ionizing) photon flux in units of photons $\mathrm{s}^{-1}$. If we evaluate the above integral between the ionization threshold energy of interest up to energies $h v_{1}=A\left(h v_{0}\right)$, where $v_{1}$ is a constant multiple of $\nu_{0}$, then the integral reduces to (switching variables to $\left.v / v_{0}\right)$ :

$$
v_{0} S_{0} \int_{1}^{A} d\left(\frac{v}{v_{0}}\right)\left(\frac{v}{v_{0}}\right)^{-(\alpha+1)}=v_{0} S_{0}\left[1-A^{-\alpha}\right], \quad \text { for } \mathrm{H} .
$$

$v_{0} S_{0} \int_{4}^{A} d\left(\frac{v}{v_{0}}\right)\left(\frac{v}{v_{0}}\right)^{-(\alpha+1)}=v_{0} S_{0}\left[4^{-\alpha}-A^{-\alpha}\right], \quad$ for He .

For H I, $h v_{0}=1$ Ryd, whereas for He II, $h v_{0}=4$ Ryd. Note that the constant $S_{0}$ through its normalization is always proportional to the product $\alpha S_{0, \mathrm{H}} /\left[v_{0}\left(1-A^{-\alpha}\right)\right]$ for $\mathrm{H}$, and to the product $\alpha S_{0, \mathrm{He}} /\left[v_{0}\left(4^{-\alpha}-A^{-\alpha}\right)\right]$ for He. When integrating up to infinity (i.e., $A=\infty$ ), the last term on the rhs simply goes to zero. Therefore, the analytic expressions for the escape fractions for $\mathrm{H}$ and $\mathrm{He}$ with source frequency dependence will respectively have the general form:

$$
f_{\mathrm{esc}, \mathrm{H}}=1-\frac{4 \pi \alpha_{\mathrm{B}, \mathrm{H}}}{\alpha S_{0, \mathrm{H}}} n_{\mathrm{e}, \mathrm{c}} n_{\mathrm{H}, \mathrm{c}}\left[\int_{0}^{\infty} \rho(r)^{2} r^{2} d r\right],
$$

and

$$
f_{\mathrm{esc}, \mathrm{He}}=1-\frac{4 \pi \alpha_{\mathrm{B}, \mathrm{He}}}{\alpha S_{0, \mathrm{He}}} n_{\mathrm{e}, \mathrm{c}} n_{\mathrm{He}, \mathrm{c}}\left[\int_{0}^{\infty} \rho(r)^{2} r^{2} d r\right],
$$

where $\rho(r)$ is the galaxy density profile. We explore several cases of $\rho(r)$ below in Section 4, but focus here on the relative values of $f_{\text {esc }}$ for $\mathrm{H}$ and $\mathrm{He}$ from frequency-dependent effects in the source spectrum. To compare with the calculation of the critical spectral index of $\alpha \sim 1.8$ derived in Section 3.1 when $\mathrm{H}$ and He photoionizations became equal, we set the second terms on the rhs of the two above equations equal to each other. This leads to:

$$
4^{-\alpha}=0.08\left(\frac{\alpha_{\mathrm{B}, \mathrm{He}}}{\alpha_{\mathrm{B}, \mathrm{H}}}\right), \quad \text { or } \alpha_{\mathrm{eff}} \simeq 0.5,
$$

for the standard values for the recombination coefficients and assuming that $n(\mathrm{He}) / n(\mathrm{H})=0.08$. Here, $\alpha_{\text {eff }}$ is the critical source spectral index after gas filtering and transmission effects are taken into account. This value of $\alpha_{\text {eff }} \sim 0.5$ is lower than the $\alpha_{\text {crit }} \sim 1.82$ derived in Section 3.1 which did not account for these effects. Both these values, however, encompass the range found in UV spectroscopic studies of low- $z$ AGNs (Zheng et al. 1997; Shull et al. 2012b; Syphers et al. 2012). 


\section{RESULTS}

Our model contains a number of parameters which control both the source spectrum and the distribution of matter around that source. In this section we explore a representative range of parameters. A summary of the results obtained can be found in Table 1.

\subsection{Gaussian Distribution Model}

We begin by considering the simple Gaussian density distribution model of Dove \& Shull (1994). We use this paper (rather than the many others cited in earlier sections) for general comparison, as its analytic results are straightforward to reproduce, at least for a pure hydrogen case. It also permits easy comparison with our results which include a number of variations in galaxy density profiles and source spectra, as well as the specific new scenarios including helium and X-rays. Adopting the infinite slab model given in Equation (7) of Dove \& Shull (1994), we assume that:

$$
n_{\mathrm{H}}(\mathbf{x})=n_{0} \exp \left(-[Z / h]^{2} / 2\right),
$$

where $n_{0}=0.312 \mathrm{~cm}^{-3}$, the Gaussian scale height $h=0.215$ $\mathrm{kpc}$, and $Z$ is the vertical distance above the midplane of the slab. The density profile for helium is the same, scaled by the cosmic helium to hydrogen ratio. We adopt a blackbody input spectrum with a temperature of $10^{4.9} \mathrm{~K}$ normalized such that the production rate of $\mathrm{H}_{\mathrm{I}}$ ionizing photons is comparable to that in the models of Pop III stars that we will consider later. Specifically, we consider a model (model "BB1" in Table 1) with ionizing photon rates $(Q)$ for $\mathrm{HI}, \mathrm{He}$ I and $\mathrm{He}$ II of, respectively, $1.4 \times 10^{51} \mathrm{~s}^{-1}, 6.1 \times 10^{50} \mathrm{~s}^{-1}$, and $2.9 \times 10^{49} \mathrm{~s}^{-1}$, and a second model (model "BB2" in Table 1 ) with the $Q$ values simply scaled up by a factor of 10 . In this model, there are relatively few $\mathrm{X}$-rays present, although we consider scenarios below with AGNs that do explicitly include them. We have also considered the contribution from high-mass X-ray binaries (HMXBs) which may form nearly simultaneously with the Pop III stars. We adopt the model of Mirabel et al. (2011) to specify the normalization of the HMXB spectrum. Specifically, their Equation (5) gives the luminosity in the $2-10 \mathrm{keV}$ range per unit star formation rate. We find that the inclusion of HMXBs makes negligible difference to the escape fractions and so do not consider them further in this work.

For these $Q$-values, the analytic formula in Equation (14) from Dove \& Shull (1994) for the critical opening angle, $\theta_{c}$, of the cone of escaping $\mathrm{H}$-ionizing radiation yields values of approximately $72^{\circ}$ and $82^{\circ}$ for the $10^{4} M_{\odot}$ and $10^{5} M_{\odot}$ Pop III clusters respectively. This is in excellent agreement with the results of the semi-analytic calculation described in Section 2, as shown in Figure 2. Specifically, there is close agreement with the angles at which $f_{\text {esc }}$ begins to drop significantly below unity, i.e., the ionizing radiation is being contained. ${ }^{7}$ The black lines in Figure 2 show the escape fraction for $\mathrm{H}$ II when we remove helium from our calculation-clearly the presence of helium causes only a small change in the escape of hydrogen-ionizing photons in this particular case.

\footnotetext{
Dove \& Shull (1994) use a Strömgren argument to determine whether any photons along a given line of sight can escape. As such, their escape fraction as a function of angle is a monotonically declining function, going from 1 to 0 at the critical angle. In our calculation we account for the non-zero mean free path of photons through the gas. This results in a smooth dependence of escape fraction on angle, making a comparison with Dove \& Shull (1994) non-trivial. Given this caveat, we find the agreement between our results and the critical angle calculation of Dove \& Shull (1994) to be acceptable.
}

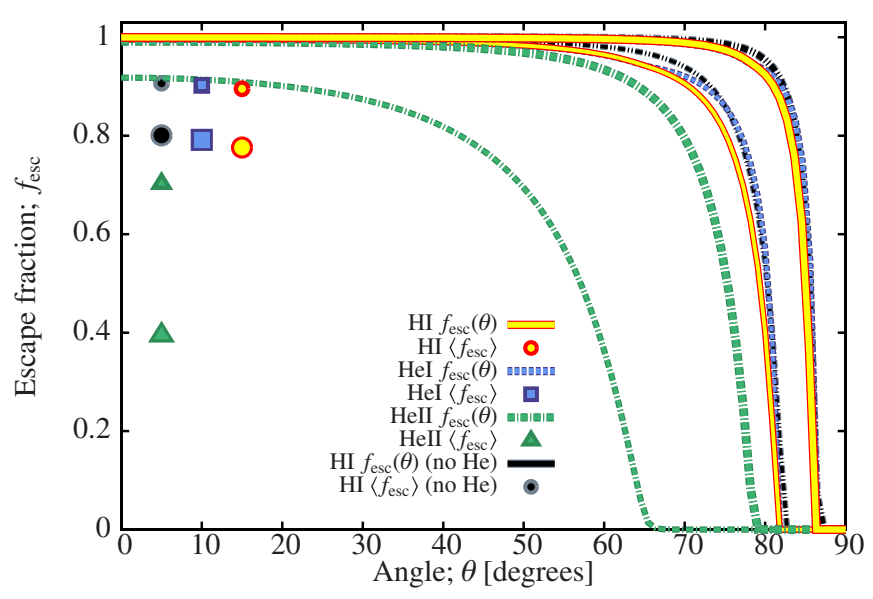

Figure 2. Escape fraction as a function of angle, $\theta$, in the Dove \& Shull (1994) Gaussian model. Thick and thin lines correspond to $10^{4} M_{\odot}$ and $10^{5} M_{\odot}$ Pop III clusters respectively. Yellow (solid), blue (dashed), and green (dotdashed) correspond to $\mathrm{HI}, \mathrm{He}$ I, and He II respectively. The net escape fractions (i.e., the escape fraction averaged over solid angle) are shown by symbols: yellow (circles), blue (squares), and green (triangles) correspond to $\mathrm{H}$ I, He I, and He II respectively. Small and large symbols corresponding to $10^{4} M_{\odot}$ and $10^{5} M_{\odot}$ Pop III clusters respectively. For comparison black (double dot-dashed) lines show the escape fraction for $\mathrm{H}$ I when no helium is included in the calculation, with the black circle showing the corresponding angle-averaged escape fraction. (A color version of this figure is available in the online journal.)

Repeating this calculation for He II and He III (with their respective recombination coefficients and $Q$-values as stated earlier), we find that for He II (He III), $\theta_{\mathrm{c}} \sim 77^{\circ}\left(11^{\circ}\right)$ and $84^{\circ}\left(63^{\circ}\right)$ for the $10^{4} M_{\odot}$ and $10^{5} M_{\odot}$ Pop III clusters. This assumes that all of the helium (for $y_{\mathrm{He}}=0.08$ ) is either in He II or He III for these $\theta_{\mathrm{c}}$ values. Comparing with the curves in Figure 2, we see that, similar to hydrogen, the $\theta_{\mathrm{c}}$ for each case corresponds to where the curves depart from their maximum value, although this is less well-defined for He III. This perhaps reveals the limitations of extending the Dove \& Shull (1994) analysis to helium, especially when we do not account for (in this simple estimate) the absorption of helium-ionizing photons by hydrogen. We do see that the critical angle for escaping He II ionizing radiation is less than that for $\mathrm{HI}$, as predicted by the analytic results earlier.

In Figure 3, we show the escape and ionization fractions as a function of radial distance from the source, evaluated at the critical angle of $\theta \sim 85^{\circ}$. Note that in Figure 3, the He II fraction rises again at large radii ( $\gtrsim 10 \mathrm{kpc})$. The density drops so rapidly at these large radii that even the weak radiation field that has escaped to this distance is able to ionize the gas. While interesting in its own regard, this is mostly irrelevant for the escape fraction calculation since the density (and optical depth) are extremely low at these large radii, especially given our constant temperature assumption. (At these low densities, cooling rates will be very low, driving the gas to higher temperatures at which collisional ionization becomes relevant.)

Last, we comment on the geometry of ionized helium versus hydrogen bubbles in the context of Dove \& Shull (1994). Beginning with their Equation (12), we can recast it as, $f_{\text {esc,i }}(\theta)=$ $1-W_{i}$, where $W_{i} \propto\left(\alpha_{i}^{(2)} n_{\mathrm{i}}\right) / Q_{i}$, here, $i$ corresponds to either $\mathrm{H}$ or $\mathrm{He}$, and the constant of proportionality is related to the product of the gas distribution and the electron density (both of which are the same for $\mathrm{H}$ and $\mathrm{He})$. The product $\left(\alpha_{i}^{(2)} n_{\mathrm{i}}\right)$ is already set for each species, and is typically less for He II and $\mathrm{He}$ III relative to $\mathrm{H}_{\mathrm{II}}$, whereas for typical Pop III clusters, $Q_{\mathrm{H}}$ 
Table 1

Escape Fractions for All Models Shown in This Work

\begin{tabular}{|c|c|c|c|c|c|c|c|c|c|c|c|c|}
\hline Profile & Source & $\begin{array}{c}M \\
\left(M_{\odot}\right)\end{array}$ & $\begin{array}{c}d \\
(\mathrm{kpc})\end{array}$ & $\mathrm{X} ?$ & $\mathrm{C} ?$ & $\begin{array}{l}Q_{0, \mathrm{H}_{\mathrm{I}}} \\
\left(\mathrm{s}^{-1}\right)\end{array}$ & $\begin{array}{c}Q_{0, \mathrm{He} \mathrm{I}} \\
\left(\mathrm{s}^{-1}\right)\end{array}$ & $\begin{array}{c}Q_{0, \mathrm{He} \mathrm{II}} \\
\left(\mathrm{s}^{-1}\right)\end{array}$ & $\begin{array}{c}f_{\mathrm{esc}, \mathrm{H} I} \\
(\%)\end{array}$ & $\begin{array}{c}f_{\mathrm{esc}, \mathrm{He} \mathrm{I}} \\
(\%)\end{array}$ & $\begin{array}{c}f_{\mathrm{esc}, \text { He II }} \\
(\%)\end{array}$ & $\begin{array}{l}\theta_{\mathrm{c}} \\
\left(^{\circ}\right)\end{array}$ \\
\hline$\beta$ & AGN & $1.0(4)$ & 0 & $\mathrm{~N}$ & $\mathrm{~N}$ & $1.17(50)$ & $6.84(49)$ & $4.10(49)$ & 1 & 2 & 0 & $\ldots$ \\
\hline$\beta$ & AGN & $1.0(4)$ & 0 & $\mathrm{~N}$ & $\mathrm{Y}$ & $1.17(50)$ & $6.84(49)$ & $4.10(49)$ & 0 & 0 & 0 & $\ldots$ \\
\hline$\beta$ & AGN & $1.0(4)$ & 0 & $\mathrm{Y}$ & $\mathrm{Y}$ & $1.17(50)$ & $6.84(49)$ & $4.10(49)$ & 10 & 18 & 30 & $\ldots$ \\
\hline$\beta$ & AGN & $1.0(4)$ & 10 & $\mathrm{~N}$ & $\mathrm{Y}$ & $1.17(50)$ & $6.84(49)$ & $4.10(49)$ & 0 & 0 & 0 & $\ldots$ \\
\hline$\beta$ & AGN & $1.0(4)$ & 10 & $\mathrm{Y}$ & $\mathrm{Y}$ & $1.17(50)$ & $6.84(49)$ & $4.10(49)$ & 12 & 20 & 33 & $\ldots$ \\
\hline$\beta$ & AGN & $1.0(6)$ & 0 & $\mathrm{~N}$ & $\mathrm{~N}$ & $2.76(52)$ & $1.14(52)$ & $4.28(51)$ & 100 & 100 & 99 & $\ldots$ \\
\hline$\beta$ & AGN & $1.0(6)$ & 0 & $\mathrm{~N}$ & $\mathrm{Y}$ & $2.76(52)$ & $1.14(52)$ & $4.28(51)$ & 99 & 99 & 97 & $\ldots$ \\
\hline$\beta$ & AGN & $1.0(6)$ & 0 & $\mathrm{Y}$ & $\mathrm{Y}$ & $2.76(52)$ & $1.14(52)$ & $4.28(51)$ & 99 & 99 & 98 & $\ldots$ \\
\hline$\beta$ & AGN & $1.0(6)$ & 10 & $\mathrm{~N}$ & $\mathrm{Y}$ & $2.76(52)$ & $1.14(52)$ & $4.28(51)$ & 99 & 99 & 97 & $\ldots$ \\
\hline$\beta$ & AGN & $1.0(6)$ & 10 & $\mathrm{Y}$ & $\mathrm{Y}$ & $2.76(52)$ & $1.14(52)$ & $4.28(51)$ & 99 & 99 & 98 & $\ldots$ \\
\hline$\beta$ & PopIII & $1.0(4)$ & 0 & $\mathrm{~N}$ & $\mathrm{~N}$ & $3.81(51)$ & $2.30(51)$ & $1.30(50)$ & 97 & 98 & 71 & $\ldots$ \\
\hline$\beta$ & PopIII & $1.0(4)$ & 0 & $\mathrm{~N}$ & $\mathrm{Y}$ & $3.81(51)$ & $2.30(51)$ & $1.30(50)$ & 93 & 95 & 32 & $\ldots$ \\
\hline$\beta$ & PopIII & $1.0(4)$ & 0 & $\mathrm{Y}$ & $\mathrm{Y}$ & $3.81(51)$ & $2.30(51)$ & $1.30(50)$ & 93 & 95 & 34 & $\ldots$ \\
\hline$\beta$ & PopIII & $1.0(4)$ & 10 & $\mathrm{~N}$ & $\mathrm{Y}$ & $3.81(51)$ & $2.30(51)$ & $1.30(50)$ & 93 & 95 & 38 & $\ldots$ \\
\hline$\beta$ & PopIII & $1.0(4)$ & 10 & $\mathrm{Y}$ & $\mathrm{Y}$ & $3.81(51)$ & $2.30(51)$ & $1.30(50)$ & 93 & 95 & 39 & $\ldots$ \\
\hline$\beta$ & PopIII & $1.0(6)$ & 0 & $\mathrm{~N}$ & $\mathrm{~N}$ & $3.81(53)$ & $2.30(53)$ & $1.30(52)$ & 100 & 100 & 100 & $\ldots$ \\
\hline$\beta$ & PopIII & $1.0(6)$ & 0 & $\mathrm{~N}$ & $\mathrm{Y}$ & $3.81(53)$ & $2.30(53)$ & $1.30(52)$ & 100 & 100 & 99 & $\ldots$ \\
\hline$\beta$ & PopIII & $1.0(6)$ & 0 & $\mathrm{Y}$ & $\mathrm{Y}$ & $3.81(53)$ & $2.30(53)$ & $1.30(52)$ & 100 & 100 & 99 & $\ldots$ \\
\hline$\beta$ & PopIII & $1.0(6)$ & 10 & $\mathrm{~N}$ & $\mathrm{Y}$ & $3.81(53)$ & $2.30(53)$ & $1.30(52)$ & 100 & 100 & 99 & $\ldots$ \\
\hline$\beta$ & PopIII & $1.0(6)$ & 10 & $\mathrm{Y}$ & $\mathrm{Y}$ & $3.81(53)$ & $2.30(53)$ & $1.30(52)$ & 100 & 100 & 99 & $\ldots$ \\
\hline$\beta$ & PopIII $^{\mathrm{a}}$ & $1.0(4)$ & 10 & $\mathrm{Y}$ & $\mathrm{Y}$ & $3.81(51)$ & $2.30(51)$ & $1.30(50)$ & 93 & 95 & 39 & $\ldots$ \\
\hline$\beta$ & PopIII $^{\mathrm{a}}$ & $1.0(6)$ & 10 & $\mathrm{Y}$ & $\mathrm{Y}$ & $3.81(53)$ & $2.30(53)$ & $1.30(52)$ & 100 & 100 & 99 & $\ldots$ \\
\hline$D \& S$ & AGN & $1.0(4)$ & $\ldots$ & $\mathrm{N}$ & $\mathrm{Y}$ & $1.17(50)$ & $6.84(49)$ & $4.10(49)$ & 31 & 35 & 23 & 45.2 \\
\hline$D \& S$ & AGN & $1.0(4)$ & $\ldots$ & $\mathrm{Y}$ & $\mathrm{Y}$ & $1.17(50)$ & $6.84(49)$ & $4.10(49)$ & 40 & 48 & 51 & 45.2 \\
\hline $\mathrm{D} \& \mathrm{~S}$ & AGN & $1.0(5)$ & $\ldots$ & $\mathrm{Y}$ & $\mathrm{Y}$ & $1.86(51)$ & $9.08(50)$ & $4.36(50)$ & 73 & 76 & 73 & 73.7 \\
\hline$D \& S$ & AGN & $1.0(6)$ & $\ldots$ & $\mathrm{N}$ & $\mathrm{Y}$ & $2.76(52)$ & $1.14(52)$ & $4.28(51)$ & 88 & 89 & 82 & 83.4 \\
\hline D\&S & AGN & $1.0(6)$ & $\ldots$ & $\mathrm{Y}$ & $\mathrm{Y}$ & $2.76(52)$ & $1.14(52)$ & $4.28(51)$ & 89 & 89 & 86 & 83.4 \\
\hline D\&S & $\mathrm{BB} 1^{\mathrm{b}, \mathrm{c}}$ & $\ldots$ & $\ldots$ & $\mathrm{Y}$ & $\mathrm{N}$ & $1.40(51)$ & $6.03(50)$ & $2.81(49)$ & 80 & 84 & 86 & 72.0 \\
\hline $\mathrm{D} \& \mathrm{~S}$ & $\mathrm{BB} 1^{\mathrm{b}, \mathrm{c}}$ & $\ldots$ & $\ldots$ & $\mathrm{Y}$ & $\mathrm{N}$ & $1.40(52)$ & $6.03(51)$ & $2.81(50)$ & 91 & 92 & 94 & 81.8 \\
\hline$D \& S$ & $\mathrm{BB} 1^{\mathrm{b}}$ & $\ldots$ & $\ldots$ & $\mathrm{Y}$ & $\mathrm{N}$ & $1.40(51)$ & $6.03(50)$ & $2.81(49)$ & 78 & 79 & 39 & 72.0 \\
\hline$D \& S$ & $\mathrm{BB} 1^{\mathrm{b}}$ & $\ldots$ & $\ldots$ & $\mathrm{Y}$ & $\mathrm{N}$ & $1.40(52)$ & $6.03(51)$ & $2.81(50)$ & 90 & 90 & 70 & 81.8 \\
\hline$D \& S$ & $\mathrm{BB} 1^{\mathrm{b}}$ & $\ldots$ & $\ldots$ & $\mathrm{Y}$ & $\mathrm{Y}$ & $1.40(52)$ & $6.03(51)$ & $2.81(50)$ & 86 & 87 & 61 & 81.8 \\
\hline $\mathrm{D} \& \mathrm{~S}$ & $\mathrm{BB} 1^{\mathrm{b}}$ & $\ldots$ & $\ldots$ & $\mathrm{Y}$ & $Y^{d}$ & $1.40(52)$ & $6.03(51)$ & $2.81(50)$ & 86 & 87 & 61 & 81.8 \\
\hline D\&S & $\mathrm{BB} 2^{\mathrm{b}}$ & $\ldots$ & $\ldots$ & $\mathrm{Y}$ & $\mathrm{Y}$ & $1.16(53)$ & $6.96(52)$ & $9.73(51)$ & 93 & 94 & 88 & 85.9 \\
\hline $\mathrm{D} \& \mathrm{~S}$ & $\mathrm{BB} 2^{\mathrm{b}}$ & $\ldots$ & $\ldots$ & $\mathrm{Y}$ & $\mathrm{Y}$ & $1.16(55)$ & $6.96(54)$ & $9.73(53)$ & 99 & 99 & 97 & 89.1 \\
\hline$D \& S$ & PopIII & $1.0(4)$ & $\ldots$ & $\mathrm{N}$ & $\mathrm{Y}$ & $3.81(51)$ & $2.30(51)$ & $1.30(50)$ & 79 & 81 & 54 & 77.2 \\
\hline$D \& S$ & PopIII & $1.0(4)$ & $\ldots$ & $\mathrm{Y}$ & $\mathrm{Y}$ & $3.81(51)$ & $2.30(51)$ & $1.30(50)$ & 79 & 81 & 54 & 77.2 \\
\hline$D \& S$ & PopIII & $1.0(5)$ & $\ldots$ & $\mathrm{Y}$ & $\mathrm{Y}$ & $3.81(52)$ & $2.30(52)$ & $1.30(51)$ & 90 & 91 & 78 & 84.1 \\
\hline$D \& S$ & PopIII & $1.0(6)$ & $\ldots$ & $\mathrm{N}$ & $\mathrm{Y}$ & $3.81(53)$ & $2.30(53)$ & $1.30(52)$ & 95 & 96 & 89 & 87.3 \\
\hline$D \& S$ & PopIII & $1.0(6)$ & $\ldots$ & $\mathrm{Y}$ & $\mathrm{Y}$ & $3.81(53)$ & $2.30(53)$ & $1.30(52)$ & 95 & 96 & 90 & 87.3 \\
\hline NFW & AGN & $1.0(4)$ & 0 & $\mathrm{~N}$ & $\mathrm{Y}$ & $1.17(50)$ & $6.84(49)$ & $4.10(49)$ & 0 & 0 & 0 & $\ldots$ \\
\hline NFW & AGN & $1.0(4)$ & 0 & $\mathrm{Y}$ & $\mathrm{Y}$ & $1.17(50)$ & $6.84(49)$ & 4.10(49) & 5 & 9 & 15 & $\ldots$ \\
\hline NFW & AGN & $1.0(4)$ & 10 & $\mathrm{~N}$ & $\mathrm{Y}$ & $1.17(50)$ & $6.84(49)$ & $4.10(49)$ & 0 & 0 & 0 & $\ldots$ \\
\hline NFW & AGN & $1.0(4)$ & 10 & $\mathrm{Y}$ & $\mathrm{Y}$ & $1.17(50)$ & $6.84(49)$ & $4.10(49)$ & 9 & 15 & 25 & $\ldots$ \\
\hline NFW & AGN & $1.0(6)$ & 0 & $\mathrm{~N}$ & $\mathrm{Y}$ & $2.76(52)$ & $1.14(52)$ & $4.28(51)$ & 94 & 94 & 79 & $\ldots$ \\
\hline NFW & AGN & $1.0(6)$ & 0 & $\mathrm{Y}$ & $\mathrm{Y}$ & $2.76(52)$ & $1.14(52)$ & $4.28(51)$ & 94 & 95 & 88 & $\ldots$ \\
\hline NFW & AGN & $1.0(6)$ & 10 & $\mathrm{~N}$ & $\mathrm{Y}$ & $2.76(52)$ & $1.14(52)$ & $4.28(51)$ & 94 & 95 & 85 & $\ldots$ \\
\hline NFW & AGN & $1.0(6)$ & 10 & $\mathrm{Y}$ & $\mathrm{Y}$ & $2.76(52)$ & $1.14(52)$ & $4.28(51)$ & 95 & 95 & 90 & $\ldots$ \\
\hline NFW & PopIII & $1.0(4)$ & 0 & $\mathrm{~N}$ & $\mathrm{Y}$ & $3.81(51)$ & $2.30(51)$ & $1.30(50)$ & 67 & 79 & 0 & $\ldots$ \\
\hline NFW & PopIII & $1.0(4)$ & 0 & $\mathrm{Y}$ & $\mathrm{Y}$ & $3.81(51)$ & $2.30(51)$ & $1.30(50)$ & 67 & 79 & 0 & $\ldots$ \\
\hline NFW & PopIII & $1.0(4)$ & 10 & $\mathrm{~N}$ & $\mathrm{Y}$ & $3.81(51)$ & $2.30(51)$ & $1.30(50)$ & 78 & 82 & 22 & $\ldots$ \\
\hline NFW & PopIII & $1.0(4)$ & 10 & $\mathrm{Y}$ & $\mathrm{Y}$ & $3.81(51)$ & $2.30(51)$ & $1.30(50)$ & 78 & 82 & 22 & $\ldots$ \\
\hline NFW & PopIII & $1.0(6)$ & 0 & $\mathrm{~N}$ & $\mathrm{Y}$ & $3.81(53)$ & $2.30(53)$ & $1.30(52)$ & 100 & 100 & 96 & $\ldots$ \\
\hline NFW & PopIII & $1.0(6)$ & 0 & $\mathrm{Y}$ & $\mathrm{Y}$ & $3.81(53)$ & $2.30(53)$ & $1.30(52)$ & 100 & 100 & 96 & $\ldots$ \\
\hline NFW & PopIII & $1.0(6)$ & 10 & $\mathrm{~N}$ & $\mathrm{Y}$ & $3.81(53)$ & $2.30(53)$ & $1.30(52)$ & 100 & 100 & 96 & $\ldots$ \\
\hline NFW & PopIII & $1.0(6)$ & 10 & $\mathrm{Y}$ & $\mathrm{Y}$ & $3.81(53)$ & $2.30(53)$ & $1.30(52)$ & 100 & 100 & 96 & $\ldots$ \\
\hline
\end{tabular}

Notes. "Profile" specifies the functional form of the density profile, while "Source" specifies the nature of the photon source, $M$ is the mass of that source, $d$ its distance from the center of the density profile. Columns "X?" and "C?" indicate whether X-rays and clouds are included respectively (see text for standard cloud model parameters). For models using the Dove \& Shull (1994) profile (labeled "D\&S"), the critical angle, $\theta_{\mathrm{c}}$, is shown in the final column. Also shown are the input ionizing fluxes, $Q_{0}$, in units of photons per second. Numbers in parentheses indicate the exponent, e.g., $1.0(4)$ means $1.0 \times 10^{4}$.

${ }^{a}$ Model spectrum includes a contribution from high-mass X-ray binaries (HMXBs).

b Model "BB1" is a blackbody source with a temperature of $7.94 \times 10^{4} \mathrm{~K}$, model "BB2" is a blackbody source with a temperature of $1.15 \times 10^{5} \mathrm{~K}$.

${ }^{c}$ Model does not include helium.

${ }^{\mathrm{d}}$ Model has cloud radii of $3 \mathrm{pc}$. 


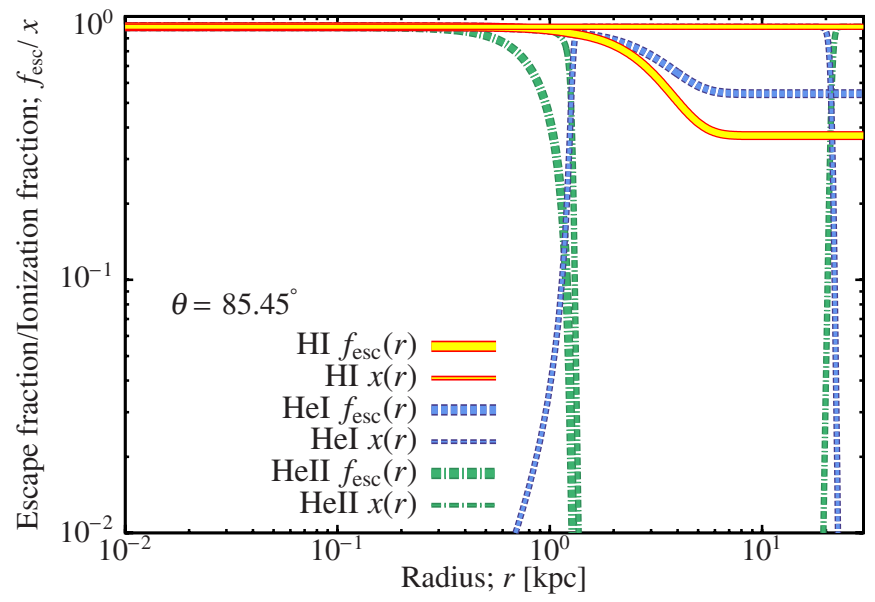

Figure 3. Escape fraction (thick lines) and ionization state (thin lines) as a function of distance from the source at $\theta=85^{\circ} .45$ (the angle at which the $\mathrm{H} \mathrm{I}$ escape fraction drops precipitously—see Figure 2), in the Dove \& Shull (1994) Gaussian model for a $10^{5} M_{\odot}$ Pop III cluster. Yellow (solid), blue (dashed), and green (dot-dashed) correspond to $\mathrm{H}$ I, He I, and He II respectively.

(A color version of this figure is available in the online journal.)

significantly exceeds $Q_{\mathrm{He}}$. Therefore, in principle, $W_{\mathrm{He}}>W_{\mathrm{H}}$, and $f_{\mathrm{esc}, \mathrm{He}}<f_{\mathrm{esc}, \mathrm{H}}$. Additionally, extending Equation (14) in Dove \& Shull (1994),

$$
\frac{\cos \left(\theta_{\mathrm{c}, \mathrm{H}}\right)}{\cos \left(\theta_{\mathrm{c}, \mathrm{He}}\right)}=\left[\frac{W_{\mathrm{H}}}{W_{\mathrm{He}}}\right]^{1 / 3},
$$

which will typically have values less than 1 , therefore implying that $\theta_{\mathrm{c}, \mathrm{He}}<\theta_{\mathrm{c}, \mathrm{H}}$. Thus, the escape of He-ionizing radiation will occur through narrower opening angles than for $\mathrm{H}$-ionizing radiation. These broad conclusions will hold in the absence of any coupling of their ionization equilibria through, e.g., X-rays or otherwise.

\subsection{Blackbody Source and Cloud Factors}

We next consider the effects of adding in clouds to our model. The left panel of Figure 4 shows the escape fraction as a function of angle for the $10^{5} M_{\odot}$ Pop III cluster model of Section 4.1 but now with a single realization ${ }^{8}$ of a population of $30 \mathrm{pc}$ radius spherical clouds with a volume filling factor of $20 \%$ and a cloud overdensity factor of $C=10$. The denser cloud regions stay more neutral and provide greater optical depth to the ionizing photons. Therefore, the escape fractions are lower than the case with no clouds.

The right panel of Figure 4 shows the radial variation of escape fractions and ionization fractions for the same model. Large fluctuations in ionization state are apparent-these are the difference between in-cloud and out-of-cloud points. The escape fraction lines show corresponding steps as the photons get strongly depleted when they encounter a cloud.

The left panel of Figure 5 shows the escape fraction as a function of angle for 10 realizations of the cloud population (thin lines) and the average over a much larger number of realizations (thick lines). Once again, we use clouds of $30 \mathrm{pc}$ radius, with a volume filling factor of $20 \%$ and $C=10$. There is significant variation in escape fraction between realizations - the angleaveraged escape fraction for He III varies from about $55 \%$ to $70 \%$ with the mean being close to $60 \%$.

To explore the effects of cloud size, the right panel of Figure 5 shows the escape fraction for the same model but now using clouds of 3 pc radius. As expected, there is much less variation in the escape fraction when using these smaller clouds-since the volume filling factor remains the same, the number of clouds is increased by a factor of 1000, reducing the fluctuation from realization to realization. The mean escape fractions are not significantly changed relative to the case of 30 pc clouds however. Note also that helium is especially sensitive to the presence of clouds, with individual realizations departing significantly from the average.

\footnotetext{
8 Note that we use the same radial distribution of clouds for each angle, $\theta$, so there are no fluctuations in escape fraction with angle. This is unrealistic of course, but this case serves only to illustrate the effects of clouds. Our primary interest is in the mean escape fraction averaged over many realizations of the cloud population, for which the lack of angular fluctuations in our calculations is irrelevant.
}

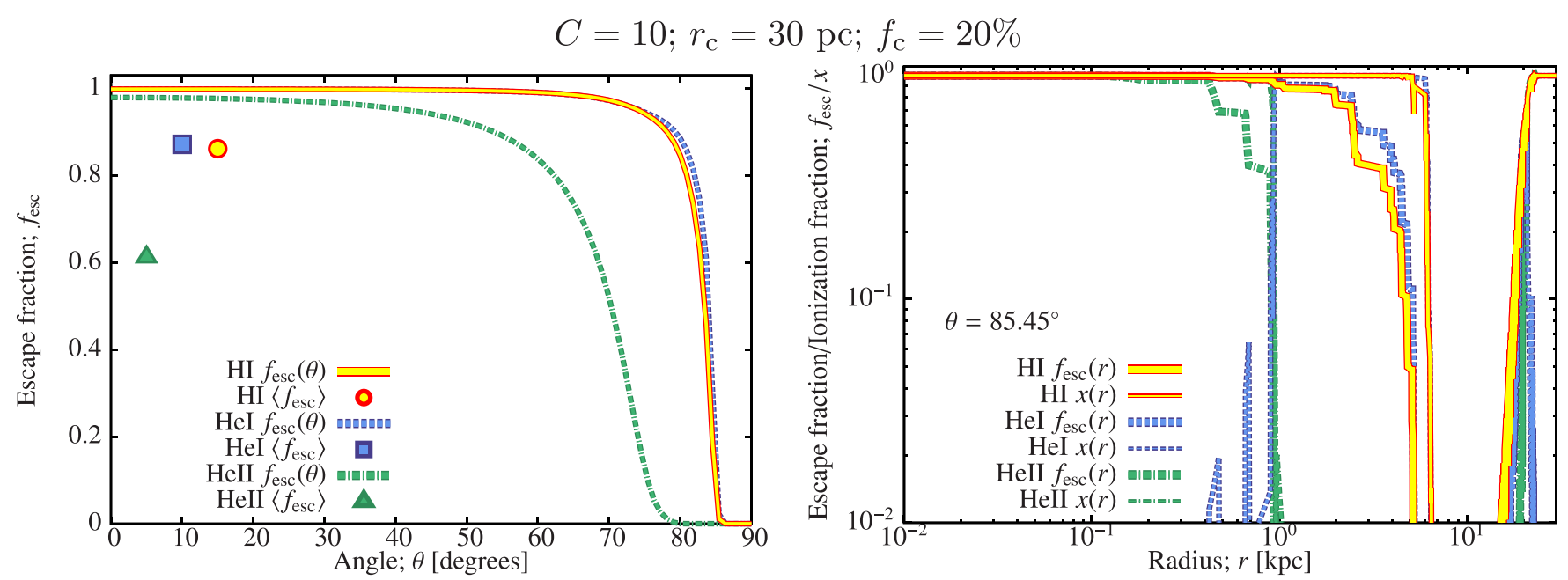

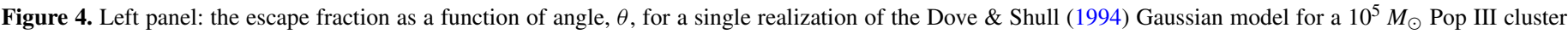

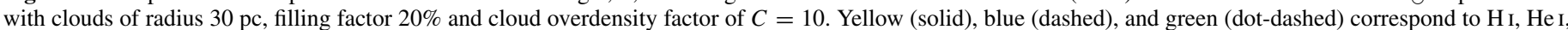

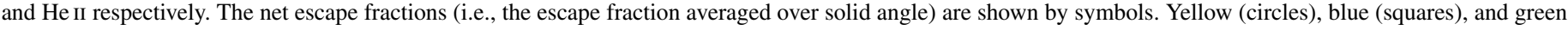

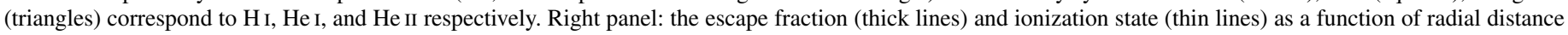
from the source at $\theta=85.45$ for the same model.

(A color version of this figure is available in the online journal.) 

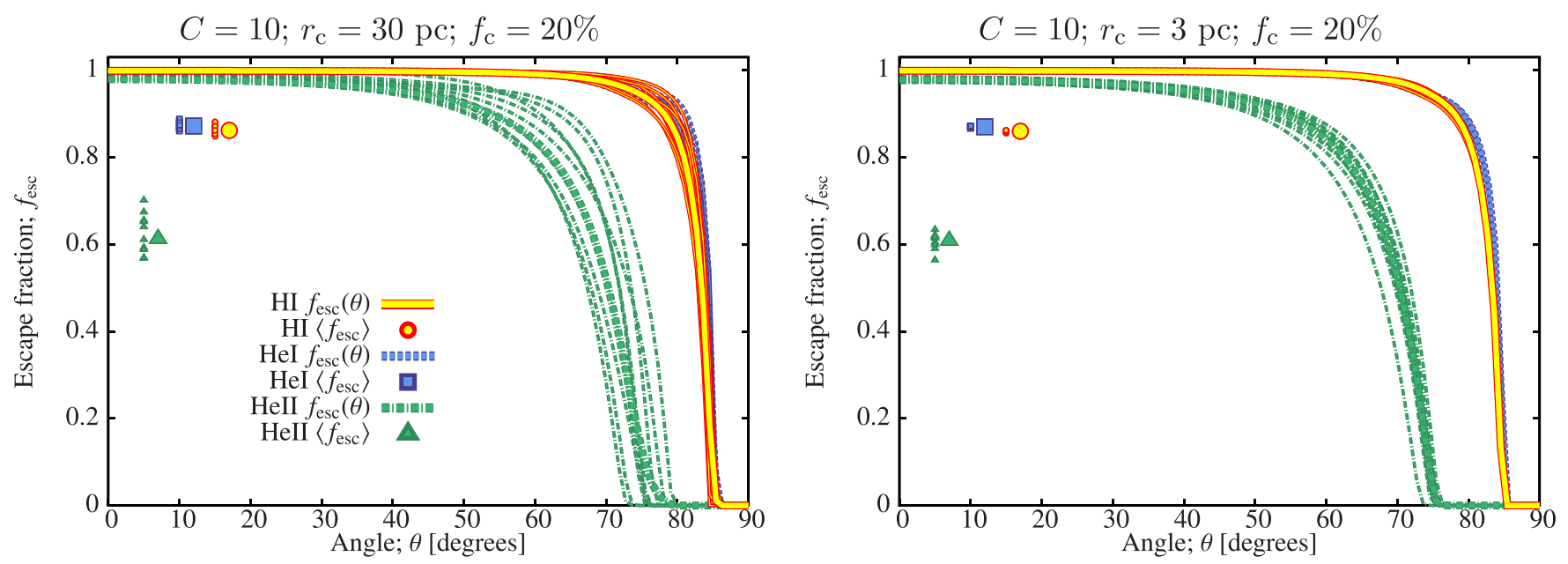

Figure 5. Left panel: the escape fraction as a function of angle, $\theta$, for 10 realizations (thin lines) and the average over many realizations (thick lines) of the Dove \& Shull (1994) Gaussian model for a $10^{5} M_{\odot}$ Pop III cluster with clouds of radius $30 \mathrm{pc}$, filling factor $20 \%$ and cloud overdensity factor of $C=10$. Same notation as Figure 4. Small symbols show mean escape fractions for individual realizations while large symbols show the mean escape fraction averaged over a large number of realizations. Right panel: the equivalent result for the same model but with clouds of radius $3 \mathrm{pc}$.

(A color version of this figure is available in the online journal.)

\subsection{Variations in Source Spectrum}

We now consider AGN and Pop III starburst cases in the Dove \& Shull (1994) Milky Way background density profile described earlier, with a cloud filling factor of $20 \%$, cloud radius $30 \mathrm{pc}$ and cloud overdensity factor of $C=$ 10. Additionally, we now include cases with and without $\mathrm{X}$-rays (in the latter of which the source spectrum is cut off above $120 \mathrm{eV}$ ). The AGN spectrum is modeled from Hopkins et al. (2007) - this gives the unabsorbed, intrinsic spectral energy distribution (SED) of AGNs as a function of their bolometric luminosity which we in turn compute from an assumed black hole mass, Eddington limit, and accretion efficiency (we assume black holes to be radiating at $10 \%$ of their Eddington luminosity). For this AGN source, we find $\left(Q_{\mathrm{HI}}, Q_{\mathrm{He}}, Q_{\mathrm{He} \text { II }}\right)=1.17,0.68,0.41 \times 10^{50}$ photons s$^{-1}$ for a $10^{4} M_{\odot}$ black hole and $\left(Q_{\mathrm{HI}}, Q_{\mathrm{He} \text { I }}, Q_{\mathrm{He} \mathrm{II}}\right)=276,114,43 \times$ $10^{50}$ photons s $^{-1}$ for a $10^{6} M_{\odot}$ black hole. The Pop III starburst spectrum is taken from Tumlinson et al. (2004), which gives $\left(Q_{\mathrm{H}}, Q_{\mathrm{He}}, Q_{\mathrm{He} \text { II }}\right)=38.10,22.97,1.30 \times 10^{50}$ photons s$^{-1}$ for a $10^{4} M_{\odot}$ burst and $\left(Q_{\mathrm{HI}}, Q_{\mathrm{He}}, Q_{\mathrm{He} \mathrm{II}}\right)=3810,2297,130 \times$ $10^{50}$ photons s $^{-1}$ for a $10^{6} M_{\odot}$ burst.

In some calculations, we place these sources in a dark matter halo of mass $10^{8} M_{\odot}$ at $z=10$. Such a halo will contain of order $10^{7} M_{\odot}$ of baryonic material. If this halo were able to convert baryons into stars as efficiently as the most efficient halos at $z=0$ (Leauthaud et al. 2012), this would imply a total stellar mass of order $10^{6} M_{\odot}$, equal to our more massive starburst model. In reality, the efficiency of both black hole and star formation is likely much lower in such a halo (due to feedback effects), so we additionally explore $10^{4} M_{\odot}$ starburst and AGN models in such a halo. Even this is arguably a very high mass for a black hole at such redshifts in a $10^{8} M_{\odot}$ halo. As such, our derived escape fractions are likely to be optimistic.

We highlight key trends in the escape fractions as a function of angle for a range of source masses, and the presence of Xrays. These trends, as shown in Figure 6 and Table 1, include the following.

1. The impact of AGNs and Pop III sources is quite different, and the effects of switching off X-rays in these two cases differ significantly, because the AGNs are more X-raydominated.
2. The difference between a blackbody spectrum (as used in Section 4.1) and these sources is substantial, owing to the $Q$-values being significantly higher (lower) for the blackbody spectrum relative to the AGNs (Pop III starburst)—compare, e.g., the $Q$ values for the $10^{4} M_{\odot}$ AGN or stellar cluster with the relevant values in Section 4.1.

3. Once the source mass exceeds about $10^{5} M_{\odot}$, the background gas (Dove \& Shull 1994) "saturates" and the escape fraction approaches unity for $\mathrm{H}$ and $\mathrm{He}$.

4. Interestingly, X-rays boost the escape fractions overall, and are particularly significant for the lower-mass AGNs or starburst masses. We also see clearly the advancing of the He III I-front relative to that of H II or He II, when X-rays are present.

In summary, the values of $f_{\text {esc }}$ found in the above subsections using the background density from Dove \& Shull (1994) are quite high, more than $80 \%-90 \%$ in some cases for $\mathrm{HI}$ and $\mathrm{He}$ I. These cases are meant to be illustrative rather than exact as we are placing a variety of sources (including primordial stars and quasars) in what is essentially a Milky Way-type disk. Some of these sources also have significantly higher $Q$-values than that assumed for a single O-type star, $10^{49} \mathrm{~s}^{-1}$, in Dove \& Shull (1994). In the following subsection we will explore primordial sources embedded in dark matter halos appropriate to the early universe.

\subsection{Variations in Galaxy Profile and/or Source Location}

Finally, we consider two alternatives to the Dove \& Shull (1994) galaxy profile: the standard Navarro-Frenk-White (NFW) profile (Navarro et al. 1997; with the gas profile appropriately scaled to the dark matter density through cosmological parameters), and the $\beta$-profile which we use to approximate the density distribution of gas in hydrostatic equilibrium at the virial temperature in an NFW halo (Ricotti \& Shull 2000). For the NFW profile, the density normalization assumes that the halo has a virial density contrast as predicted by the spherical top-hat collapse model for our chosen cosmology $\left(\Omega_{\mathrm{M}}=0.275\right.$, $\Omega_{\Lambda}=0.725$; Komatsu et al. 2011), and the scale radius is set using the fitting function of Gao et al. (2008). For the $\beta$-profile, we set parameters using the results of Ricotti \& Shull (2000) 
DS94 profile; $10^{4} M_{\odot}$ AGN; X-rays included

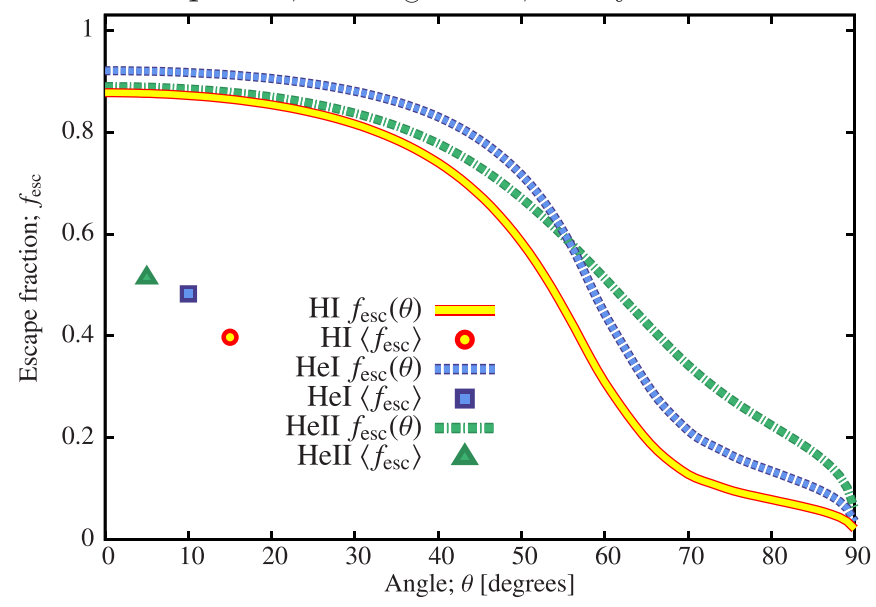

DS94 profile; $10^{4} M_{\odot}$ AGN; X-rays excluded

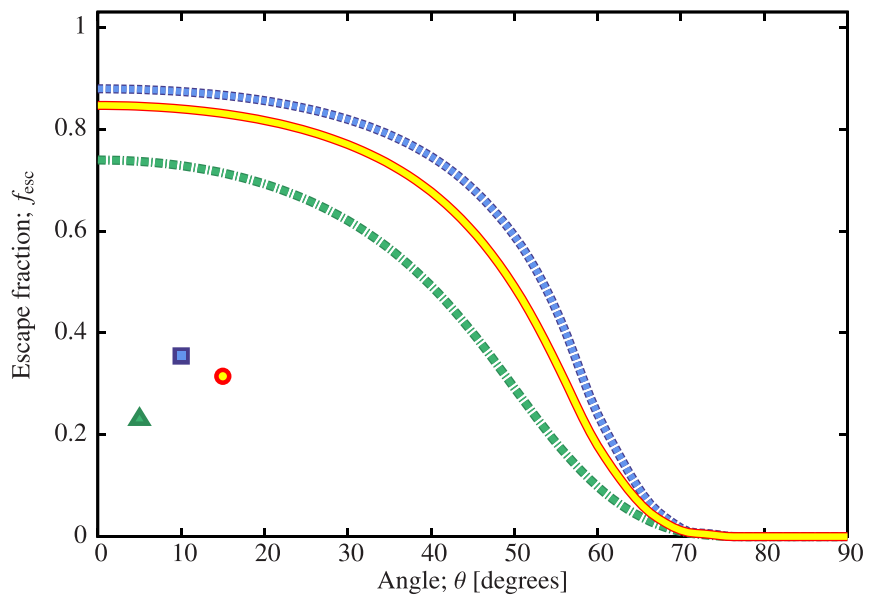

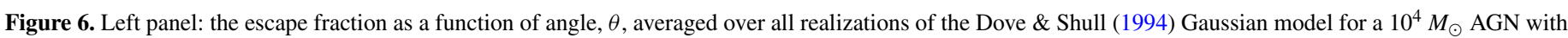

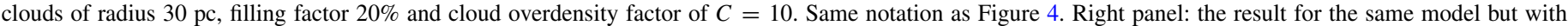
$\mathrm{X}$-rays removed from the SED.

(A color version of this figure is available in the online journal.)

$\beta$-profile; $10^{4} M_{\odot}$ AGN; X-rays included

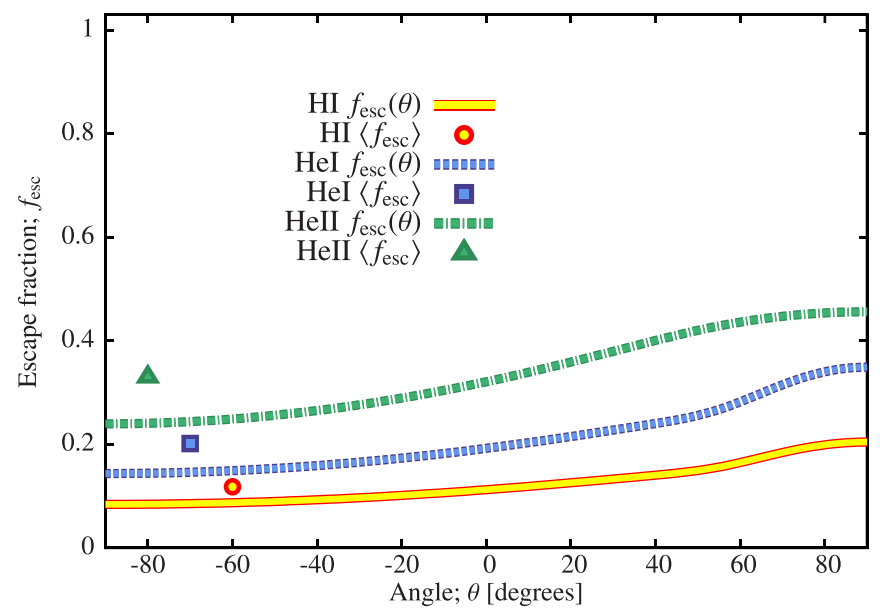

$\beta$-profile; $10^{4} M_{\odot} \mathrm{AGN} ; \mathrm{X}$-rays excluded

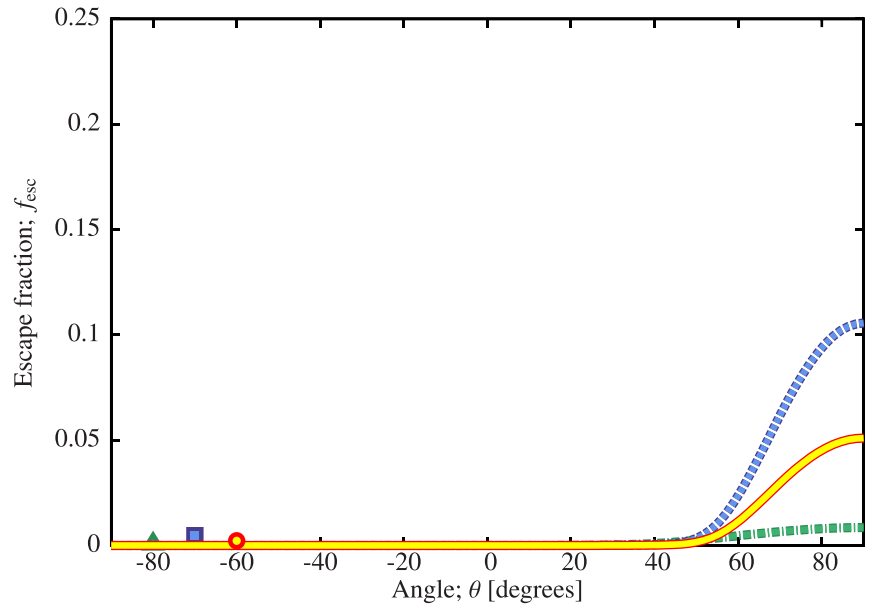

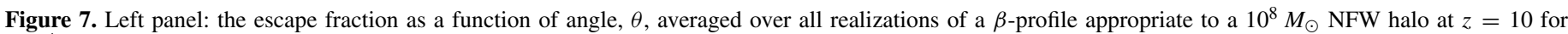

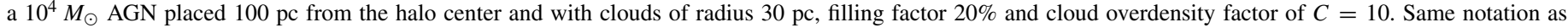
Figure 4. Right panel: the result for the same model but with X-rays removed from the SED.

(A color version of this figure is available in the online journal.)

such that the profile approximates that of gas in hydrostatic equilibrium in an NFW density profile.

We explored cases for a range of galaxy dark matter masses but display here the results for a $10^{8} M_{\odot}$ halo, whose virial radius is about 1.4 (physical) $\mathrm{kpc}$ at $z=10$. In the Dove \& Shull (1994) model used in earlier sections, the density profile drops away from the source as a Gaussian; thus, the escape fraction quickly reaches a constant value with distance from the source. In the case of an NFW or $\beta$-profile, however, the density falls off only as $r^{-3}$ on large scales, so the escape fraction converges more slowly. We therefore use the virial radius of the halo as the radius at which to stop integrating and measure the escape fraction. The virial radius marks the dividing line between the galactic environment and the circumgalactic medium (CGM). Therefore, any photons escaping beyond the virial radius are able to ionize the CGM, in keeping with the usual definition of the escape fraction.

For these two density profiles (NFW and $\beta$ ), we consider cases where the source is at the center, and cases where the source is placed $100 \mathrm{pc}$ from the halo center $(\sim 10 \%$ of the halo virial radius). The figures displayed in this section show the escape fraction at the virial radius as a function of angle, as in earlier sections of the paper. For the off-center source cases, note that $\theta=-90^{\circ}\left(\theta=+90^{\circ}\right)$ is the direction from the source directly through (directly away from) the center of the halo, and, consequently, always has the lowest (highest) escape fraction. The angle $\theta=0^{\circ}$ corresponds to directions perpendicular to the halo center from the source. Most of the solid angle is around $\theta=0^{\circ}$, so this typically gives the best estimate of the mean escape fraction.

Considering first the case of a $\beta$-profile with a $10^{4} M_{\odot}$ mass AGN black-hole source (Figure 7), there is a large difference when X-rays are excluded from the AGN SED, indicating that it is primarily the X-rays that are able to penetrate the halo and escape into the IGM. Furthermore, in the case including X-rays, it is the He II continuum photons (energies $\geqslant 4$ Ryd) which have the highest escape fraction, due to the low cross-section for photons at these energies. For the $10^{4} M_{\odot}$ Pop III starburst 

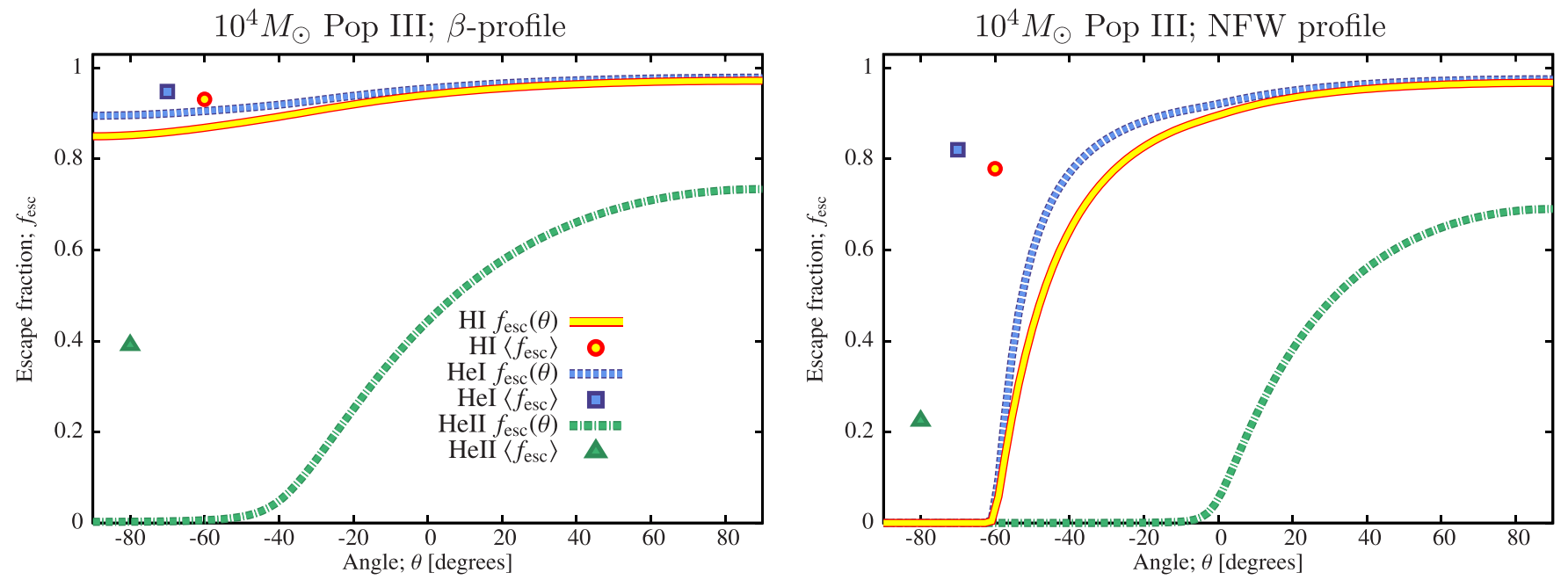

Figure 8. Left panel: the escape fraction as a function of angle, $\theta$, averaged over all realizations of a $\beta$-profile appropriate to a $10^{8} M_{\odot}$ NFW halo at $z=10$ for a $10^{4} M_{\odot}$ Pop III cluster placed $100 \mathrm{pc}$ from the halo center and with clouds of radius $30 \mathrm{pc}$, filling factor $20 \%$ and cloud overdensity factor of $C=10$. Same notation as Figure 4. Right panel: the result for the same model but using the NFW itself rather than the corresponding $\beta$-profile.

(A color version of this figure is available in the online journal.)

$\beta$-profile; $10^{4} M_{\odot}$ AGN; X-rays included

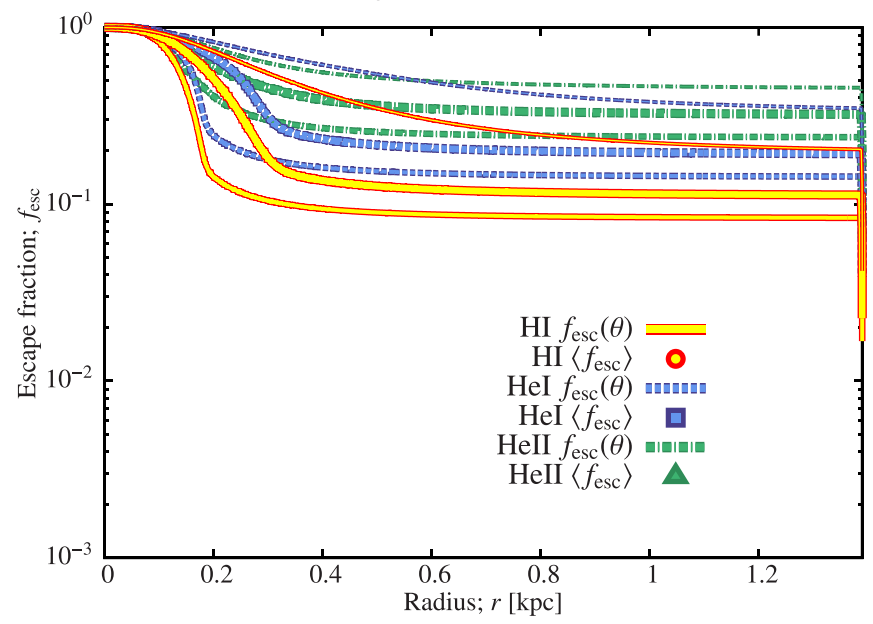

$\beta$-profile; $10^{4} M_{\odot}$ AGN; X-rays excluded

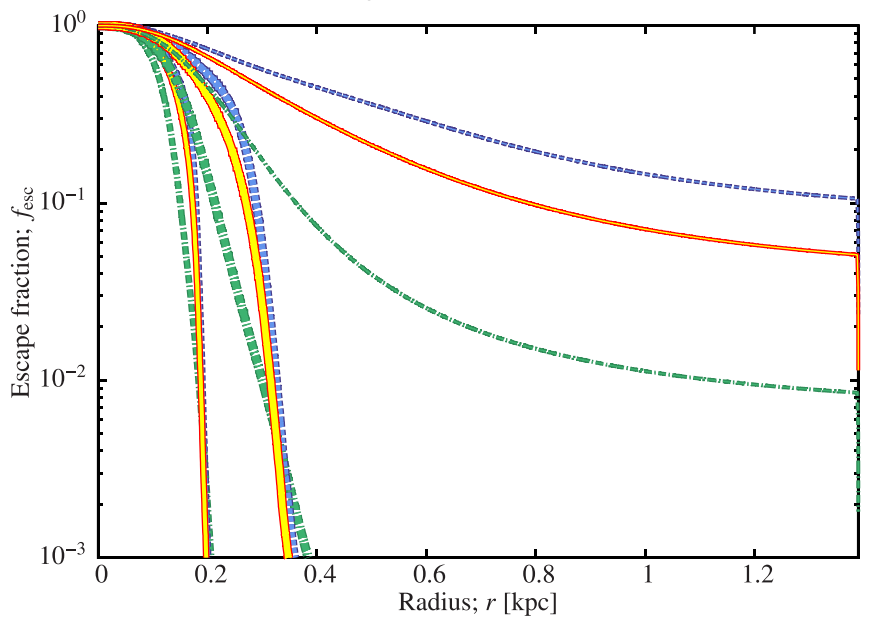

Figure 9. Left panel: the escape fraction (thick lines) and ionization fraction (thin lines) as a function of distance, $r$, from the source, averaged over all realizations of a $\beta$-profile appropriate to a $10^{8} M_{\odot}$ NFW halo at $z=10$ for a $10^{4} M_{\odot}$ AGN placed $100 \mathrm{pc}$ from the halo center and with clouds of radius $30 \mathrm{pc}$, filling factor $20 \%$ and cloud overdensity factor of $C=10$. Same notation as Figure 4. Right panel: the result for the same model but with X-rays removed from the SED.

(A color version of this figure is available in the online journal.)

(Figure 8$), f_{\text {esc }}$ is nearly unity for $\mathrm{HI}$ and $\mathrm{He}$ II ionizing radiation for all of the escape directions for a $\beta$-profile. The $f_{\text {esc }}$ values for He II ionizing radiation is significantly lower for all angles, approaching zero toward the direction of the galaxy center. If the source is instead placed precisely at the halo center, the escape fraction (which is now independent of angle due to the spherical symmetry) corresponds to that at $\theta=-15^{\circ}$ in Figure 8 .

In the cases of the $10^{6} M_{\odot} \mathrm{AGN}$, the values of $f_{\text {esc }}$ saturate quickly to 1 for all species, except for the NFW cases near the very core (owing to the high densities there). X-rays make a difference in the direction of center but not otherwise. Hence, we do not display them, although here too, the He curve gets ahead of $\mathrm{H}$ in the very core of the galaxy. We find similar results for the case of the $10^{6} M_{\odot}$ Pop III starburst, except that there is very little difference with X-rays included owing to the low $\mathrm{X}$-ray contribution to the SED in this case.

Figure 9 shows the dependence of escape fraction on radial distance for a $10^{4} M_{\odot}$ AGN source placed $100 \mathrm{pc}$ from the center of our $\beta$-profile model. The radial dependence is shown along three different directions-directly away from the halo center, perpendicular to the direction to the halo center, and directly through the halo center. In the left panel, which includes $\mathrm{X}$-rays, the escape fractions quickly asymptote to almost constant values of between $10 \%$ and $60 \%$ in all directions. The right panel of Figure 9 shows the same calculation but with X-rays removed from the source SED. The behavior is substantially different in this case. In directions through the halo center and perpendicular to that direction the escape fractions plummet rapidly, indicating that it was the X-rays which were able to escape through these directions most easily. Directly away from the center, the escape fractions remain significant, but continue to fall out to the halo's virial radius.

In summary for this subsection, we find that an NFW gas distribution produces much lower escape fractions overall for both $\mathrm{H}$ and $\mathrm{He}$ relative to the $\beta$-profile, particularly through the halo center where the density rises rapidly. Additionally, 
the presence of X-rays boosts $f_{\text {esc }}$ for $\mathrm{HI}$, He I and He II; this is especially dramatic for He II.

\section{CONCLUSIONS AND DISCUSSION}

We have calculated the escape fraction of ionizing radiation, $f_{\text {esc }}$, for both hydrogen and helium using one-dimensional semianalytic models of galaxies ranging from those typical of the early universe to disk-type galaxies that are not heavily dustobscured. We have considered many scenarios that vary the galaxy density profile, the source type (first stars or AGNs), location, and spectral index, as well as cloud overdensity factors. Key new points in our work include calculations of $f_{\text {esc }}$ for $\mathrm{He} \mathrm{I}$ and $\mathrm{He} \mathrm{II}$ ionizing radiation, and the inclusion of the process of partial gas ionization by X-rays. We find that X-rays play an important role in boosting the $f_{\text {esc }}$ values for $\mathrm{HI}, \mathrm{He}$ I and He II, especially He II. Escape fractions from primordial halos containing a bright AGN may reach as high as 30\%:20\%:10\% for H II:He II:He III (mostly due to the significant contribution from X-rays in such sources), while a burst of Pop III star formation could result in escape fractions as high as 90\%:90\%:40\% for H II:He II:He III. For sufficiently hard firstlight sources, the helium I-fronts closely track or even advance beyond that of hydrogen.

These calculations have an impact on a number of different observational probes of first-light sources and reionization models. First, the current Wilkinson Microwave Anisotropy Probe 9 results indicate that the Thomson optical depth, once covariances are accounted for, is $\tau_{e} \sim 0.08-0.09$ (Hinshaw et al. 2012), allowing for an extended period of partial ionization of $\mathrm{H}$ and/or He beyond $z \sim 6$. As discussed in Shull \& Venkatesan (2008), the need for an extreme reionization scenario is reduced for $\mathrm{H}$, if $f_{\mathrm{esc}, \mathrm{He}}$ is competitive with $f_{\mathrm{esc}, \mathrm{H}}$. With primordial helium at $Y=0.25$ by mass, the number ratio $y=n_{\mathrm{He}} / n_{\mathrm{H}}=$ $1 / 12$. The inclusion of helium in models typically increases $\tau_{e}$ by $16 \%$ relative to that for hydrogen only (Shull \& Venkatesan 2008), accounting for singly ionized helium at $3 \leqslant z \leqslant 7$ and doubly ionized helium at $z<3$.

Second, the values of $f_{\text {esc }}$ and its redshift evolution can impact the evolution of the optical depth of He II and the ratio of He III to He II at $z \sim 2.5-3$ (Khaire \& Srianand 2012; Shull et al. 2012b), during late He II reionization. The ratio of He II to H I column densities, denoted by $\eta$, has been observed to have factor-of-ten variations on megaparsec scales, along the sightlines to several "He-II quasars" at $z \sim 2.4-2.9$ (Shull et al. 2004, 2010; Fechner et al. 2006; Syphers et al. 2012). Because He II is photoionized by 4 Ryd continuum radiation, these $\eta$ variations are probably dominated by fluctuations in the intrinsic spectra of the AGN sources, modulated by He II opacity changes in the IGM (primarily from Lyman-limit systems). Ultraviolet spectroscopic studies of low-redshift AGNs (Zheng et al. 1997; Shull et al. 2012b) find rest-frame (1-2 Ryd) ionizing spectra, $F_{v} \propto v^{-\alpha}$, with a wide range of spectral indices from hard $(\alpha \approx 0.5)$ to soft ( $\alpha \approx 3$ ). This is consistent with the range of the critical spectral index of $\alpha \sim 0.5-1.8$ derived in Section 3 when accounting for frequency-dependent effects in the source spectrum. The composite EUV spectra of low-redshift AGNs have recently been measured to have $\langle\alpha\rangle \approx 1.4-1.5$ between 1.0 and 1.5 Ryd (Shull et al. 2012b).

Last, calculations of $f_{\text {esc }}$ impact the converse problem of radiation trapped in primordial galaxies, and have consequences for detecting these galaxies through emission-line signatures. Such emission-line signatures of very hot, low metallicity massive stars (Tumlinson \& Shull 2000; Tumlinson et al. 2001) include strong He II recombination lines such as $\lambda 1640$ and $\lambda 4686$.

Looking ahead, science drivers for future UV spectroscopic missions (Tumlinson et al. 2012; McCandliss et al. 2012) include telescopes and spectrographs with spectral coverage down to the hydrogen Lyman limit ( $912 \AA)$. This coverage will allow one to directly measure escaping (hydrogen) LyC radiation from low-redshift starburst galaxies. This far-UV band also probes He II Gunn-Peterson absorption between $z \approx 2-3$, an interval that spans the patchy He II post-reionization epoch in the restframe of the intervening IGM (Shull et al. 2004, 2010; Syphers \& Shull 2013). Observations of "He II quasars" at somewhat higher redshifts, $z=3.0-3.5$ (Syphers et al. 2009a, 2009b, 2012; Worseck et al. 2011) can observe the He II absorption during the epochs when the He III ionized bubbles have not yet overlapped. Placing direct limits on $f_{\text {esc,He }}$ faces significant observational challenges, however, and the likelihood of a FUV instrument as a successor to COS remains uncertain at present.

We thank Jason Tumlinson for useful correspondence on the spectra from metal-free stellar populations, and Yue Zhao, Yang Wang, and Long Yan Yung for useful input. A.V. gratefully acknowledges support from Research Corporation through the Single Investigator Cottrell College Science Award, and from the University of San Francisco Faculty Development Fund. J.M.S. acknowledges support from NASA through Astrophysics Theory grant NNX07-AG77G.

\section{REFERENCES}

Aver, E., Olive, K. A., \& Skillman, E. D. 2010, JCAP, 05, 003

Becker, G. D., Bolton, J. S., Haehnelt, M. G., \& Sargent, W. L. W. 2011, MNRAS, 410, 1096

Benson, A. J., Nusser, A., Sugiyama, N., \& Lacey, C. G. 2001, MNRAS, 320,153

Bromm, V., Kudritzki, R. P., \& Loeb, A. 2001, ApJ, 552, 464

Ciardi, B., Bolton, J. S., Maselli, A., \& Graziani, L. 2012, MNRAS, 423, 558

Deharveng, J.-M., Buat, V., Le Brun, V., et al. 2001, A\&A, 375, 805

Donahue, M., \& Shull, J. M. 1991, ApJ, 383, 511

Dove, J. B., \& Shull, J. M. 1994, ApJ, 430, 222

Dove, J. B., Shull, J. M., \& Ferrara, A. 2000, ApJ, 531, 846

Dunlop, J. S., Rogers, A. B., McLure, R. J., et al. 2012, arXiv:1212.0860

Ellis, R. S., McLure, R. J., Dunlop, J. S., et al. 2013, ApJL, 763, L7

Fechner, C., Reimers, D., Kriss, G. A., et al. 2006, A\&A, 455, 91

Fernandez, E. R., \& Shull, J. M. 2011, ApJ, 731, 20

Finkelstein, S. L., Papovich, C., Ryan, J. R. E., et al. 2012, ApJ, 758, 93

Gao, L., Navarro, J. F., Cole, S., et al. 2008, MNRAS, 387, 536

Gnedin, N. Y., Kravtsov, A. V., \& Chen, H.-W. 2008, ApJ, 672, 765

Grimes, J. P., Heckman, T., Aloisi, A., et al. 2009, ApJS, 181, 272

Heckman, T. M., Borthakur, S., Overzier, R., et al. 2011, ApJ, 730, 5

Hinshaw, G., Larson, D., Komatsu, E., et al. 2012, ApJS, submitted, arXiv: 1212.5226

Hopkins, P. F., Richards, G. T., \& Hernquist, L. 2007, ApJ, 654, 731

Khaire, V., \& Srianand, R. 2013, MNRAS, 431, 53

Komatsu, E., Smith, K. M., Dunkley, J., et al. 2011, ApJS, 192, 18

Leauthaud, A., Tinker, J., Bundy, K., et al. 2012, ApJ, 744, 159

Leitherer, C., Ferguson, H. C., Heckman, T. M., \& Lowenthal, J. D. 1995, ApJL, 454, L19

Loeb, A., \& Barkana, R. 2001, ARA\&A, 39, 19

McCandliss, S. R., Andersson, B.-G., Bergvall, N., et al. 2012, arXiv:1209.3320

McLure, R. J., Dunlop, J. S., Bowler, R. A. A., et al. 2013, MNRAS, in press (arXiv:1212.5222)

Mirabel, I. F., Dijkstra, M., Laurent, P., Loeb, A., \& Pritchard, J. R. 2011, A\&A, 528,149

Navarro, J. F., Frenk, C. S., \& White, S. D. M. 1997, ApJ, 490, 493

Nestor, D. B., Shapley, A. E., Steidel, C. C., \& Siana, B. 2011, ApJ, 736, 18

Peimbert, M., Luridiana, V., \& Peimbert, A. 2007, ApJ, 666, 636

Razoumov, A. O., \& Sommer-Larsen, J. 2010, ApJ, 710, 1239

Rhoads, J. E., Xu, C., Dawson, S., et al. 2004, ApJ, 611, 59

Ricotti, M., Gnedin, N. Y., \& Shull, J. M. 2002, ApJ, 575, 49

Ricotti, M., \& Shull, J. M. 2000, ApJ, 542, 548 
Robertson, B. E., Furlanetto, S. R., Schneider, E., et al. 2013, ApJ, 768, 71

Schenker, M. A., Robertson, B. E., Ellis, R. S., et al. 2013, ApJ, 768, 196

Shapley, A. E., Steidel, C. C., Pettini, M., Adelberger, K. L., \& Erb, D. K. 2006, ApJ, 651, 688

Shull, J. M., France, K., Danforth, C. W., Smith, B., \& Tumlinson, J. 2010, ApJ, 722,1312

Shull, J. M., Harness, A., Trenti, M., \& Smith, B. D. 2012a, ApJ, 747,100

Shull, J. M., Stevans, M., \& Danforth, C. W. 2012b, ApJ, 752, 162

Shull, J. M., Tumlinson, J., Giroux, M. L., Kriss, G. A., \& Reimers, D. 2004, ApJ, 600,570

Shull, J. M., \& Venkatesan, A. 2008, ApJ, 685, 1

Siana, B., Teplitz, H. I., Ferguson, H. C., et al. 2010, ApJ, 723, 241

Steidel, C. C., Pettini, M., \& Adelberger, K. L. 2001, ApJ, 546, 665

Syphers, D., Anderson, S. F., Zheng, W., et al. 2009a, ApJ, 690, 1181
Syphers, D., Anderson, S. F., Zheng, W., et al. 2009b, ApJS, 185, 20

Syphers, D., Anderson, S. F., Zheng, W., et al. 2012, AJ, 143, 100

Syphers, D., \& Shull, J. M. 2013, ApJ, 765, 119

Tumlinson, J., Aloisi, A., Kriss, G., et al. 2012, arXiv:1209.3272

Tumlinson, J., Giroux, M. L., \& Shull, J. M. 2001, ApJL, 550, L1

Tumlinson, J., \& Shull, J. M. 2000, ApJL, 528, L65

Tumlinson, J., Shull, J. M., \& Venkatesan, A. 2003, ApJ, 584, 608

Tumlinson, J., Venkatesan, A., \& Shull, J. M. 2004, ApJ, 612, 602

Venkatesan, A., Giroux, M. L., \& Shull, J. M. 2001, ApJ, 563, 1

Wise, J. H., \& Cen, R. 2009, ApJ, 693, 984

Wood, K., \& Loeb, A. 2000, ApJ, 545, 86

Worseck, G., Prochaska, J. X., McQuinn, M., et al. 2011, ApJL, 733, L24

Yajima, H., Choi, J.-H., \& Nagamine, K. 2011, MNRAS, 412, 411

Zheng, W., Kriss, G. A., Telfer, R. C., Grimes, J. P., \& Davidsen, A. F. 1997, ApJ, 475,469 